\title{
The virtual observatory service TheoSSA: Establishing a database of synthetic stellar flux standards
}

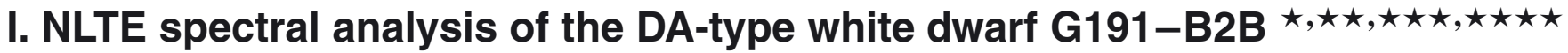

\author{
T. Rauch ${ }^{1}$, K. Werner ${ }^{1}$, R. Bohlin ${ }^{2}$, and J. W. Kruk ${ }^{3}$ \\ ${ }^{1}$ Institute for Astronomy and Astrophysics, Kepler Center for Astro and Particle Physics, Eberhard Karls University, Sand 1, \\ 72076 Tübingen, Germany, \\ e-mail: rauch@astro.uni-tuebingen.de \\ 2 Space Telescope Science Institute, 3700 San Martin Drive, Baltimore MD 21218, USA \\ 3 NASA Goddard Space Flight Center, Greenbelt MD 20771, USA
}

Received 22 July 2013 / Accepted 22 August 2013

\section{ABSTRACT}

\begin{abstract}
Context. Hydrogen-rich, DA-type white dwarfs are particularly suited as primary standard stars for flux calibration. State-of-the-art NLTE models consider opacities of species up to trans-iron elements and provide reliable synthetic stellar-atmosphere spectra to compare with observations.

Aims. We will establish a database of theoretical spectra of stellar flux standards that are easily accessible via a web interface.

Methods. In the framework of the Virtual Observatory, the German Astrophysical Virtual Observatory developed the registered service TheoSSA. It provides easy access to stellar spectral energy distributions (SEDs) and is intended to ingest SEDs calculated by any model-atmosphere code. In case of the DA white dwarf G191-B2B, we demonstrate that the model reproduces not only its overall continuum shape but also the numerous metal lines exhibited in its ultraviolet spectrum.

Results. TheoSSA is in operation and contains presently a variety of SEDs for DA-type white dwarfs. It will be extended in the near future and can host SEDs of all primary and secondary flux standards. The spectral analysis of G191-B2B has shown that our hydrostatic models reproduce the observations best at $T_{\text {eff }}=60000 \pm 2000 \mathrm{~K}$ and $\log g=7.60 \pm 0.05$. We newly identified Fe VI, Ni VI, and $\mathrm{Zn}$ IV lines. For the first time, we determined the photospheric zinc abundance with a logarithmic mass fraction of $-4.89(7.5 \times$ solar). The abundances of $\mathrm{He}$ (upper limit), C, N, O, Al, Si, O, P, S, Fe, Ni, Ge, and $\mathrm{Sn}$ were precisely determined. Upper abundance limits of about $10 \%$ solar were derived for $\mathrm{Ti}, \mathrm{Cr}, \mathrm{Mn}$, and $\mathrm{Co}$.

Conclusions. The TheoSSA database of theoretical SEDs of stellar flux standards guarantees that the flux calibration of all astronomical data and cross-calibration between different instruments can be based on the same models and SEDs calculated with different model-atmosphere codes and are easy to compare.
\end{abstract}

Key words. standards - stars: abundances - stars: atmospheres - stars: individual: G191-B2B - virtual observatory tools white dwarfs

\section{Introduction}

In the framework of the Virtual Observatory (VO), the German Astrophysical Virtual Observatory (GAVO) project provides synthetic stellar spectra on demand via the registered Theoretical Stellar Spectra Access (TheoSSA) VO service (Rauch 2008a; Rauch \& Nickelt 2009; Rauch et al. 2009). These SEDs can be used for spectral analyses (Rauch et al. 2010; Ringat \& Rauch 2010; Rauch \& Ringat 2011; Ringat et al. 2012) or serve as

\footnotetext{
* Based on observations with the NASA/ESA Hubble Space Telescope, obtained at the Space Telescope Science Institute, which is operated by the Association of Universities for Research in Astronomy, Inc., under NASA contract NAS5-26666.

$\star \star$ Based on observations made with the NASA-CNES-CSA Far Ultraviolet Spectroscopic Explorer.

$\star \star \star$ Figures 1, 6, 10-12, 23, A.1, A.2 and Tables 2-4 are available in electronic form at http://www . aanda.org

$\star \star \star \star$ Table 5 and Figs. A.1 and A.2 (FITS files) are only available at the CDS via anonymous ftp to

cdsarc.u-strasbg. fr (130.79.128.5) or via

http://cdsarc.u-strasbg.fr/viz-bin/qcat?]/A+A/560/A106
}

ionizing spectra for e.g. photoionization models of ionized nebulae. The registered TMAW VO tool ${ }^{1}$, that allows to calculate individual NLTE model atmospheres considering opacities of $\mathrm{H}, \mathrm{He}, \mathrm{C}, \mathrm{N}, \mathrm{O}, \mathrm{Ne}, \mathrm{Na}$, and $\mathrm{Mg}$, provides additional SEDs which are automatically ingested by TheoSSA. Figure 1 shows the complete action scheme for a VO user to retrieve an SED.

With the increasing usage of TheoSSA over the last years, it became necessary to demonstrate the reliability of the SEDs. We established simple benchmark tests (Ringat et al. 2012) to show the achievable analysis precision, e.g. in the determination of effective temperatures $\left(T_{\text {eff }}\right)$ and surface gravities $(\log g)$, in cases that TMAW SEDs are used which are calculated with standard model atoms that are limited in the number of atomic levels treated in NLTE. This guarantees model calculations in a reasonable time for a $\mathrm{VO}$ user.

Since 2012 TheoSSA also includes synthetic spectra of spectrophotometric standard stars. In this paper, we start to systematically establish a database of these and address the reliability

\footnotetext{
Tübingen Model-Atmosphere WWW Interface, http://astro. uni-tuebingen. de/ TMAW
} 
Table 1. Parameters of the HST DA standard stars (Gianninas et al. 2011).

\begin{tabular}{lccc}
\hline \hline Name & WD no. $^{a}$ & $\begin{array}{c}T_{\text {eff }} \\
{[\mathrm{K}]}\end{array}$ & $\begin{array}{c}\log g \\
{\left[\mathrm{~cm} / \mathrm{s}^{2}\right]}\end{array}$ \\
\hline G191-B2B & $0501+527$ & $60920 \pm 993$ & $7.55 \pm 0.05$ \\
GD 71 & $0549+158$ & $33590 \pm 483$ & $7.93 \pm 0.05$ \\
GD 153 & $1254+223$ & $40320 \pm 626$ & $7.93 \pm 0.05$ \\
HZ 43A $^{b}$ & $1314+293$ & $56800 \pm 1249^{c}$ & $7.89 \pm 0.07$ \\
\hline
\end{tabular}

Notes. ${ }^{(a)}$ WD numbers are from McCook \& Sion (1999). ${ }^{(b)}$ HZ 43A is only used in the UV because of contamination at longer wavelengths from its M-dwarf companion. ${ }^{(c)}$ Beuermann et al. $(2006,2008)$ determined $T_{\text {eff }}=51111 \pm 660 \mathrm{~K}$ and $\log g=7.90 \pm 0.080$.

of state-of-the-art model-atmosphere spectra and the achievable limits in future flux calibration.

White dwarfs (WDs) are ideal objects for the calibration of astronomical observations (Rauch 2012). They are relatively simple objects and their radiation is determined by fundamental physics, e.g. their radius is defined by electron degeneracy. Moreover, they are nearby and their distance can be measured precisely, at least by the upcoming GAIA ${ }^{2}$ mission (cf. Pancino et al. 2012, for a description of the GAIA spectrophotometric standard stars survey). Most of the hot, hydrogen-rich WDs (spectral type DA) with $T_{\text {eff }}<40000 \mathrm{~K}$ have virtually pure hydrogen atmospheres (gravitational settling), while the hotter WDs exhibit lines of heavier elements due to radiative levitation. WD spectral modeling requires adequate observations (WDs are intrinsically faint) and state-of-the-art theoretical model atmospheres that account for reliable physics and deviations from local thermodynamic equilibrium (LTE).

The hot DA-type WD G191-B2B (BD+52 913$)$ is, together with GD 71, and GD 153, one of the primary flux reference standards for all absolute calibrations from 1000 to $25000 \AA$ (Bohlin 2007). Recent results for their $T_{\text {eff }}$ and $\log g$ are summarized in Table 1. G191-B2B, the hottest and visually brightest $\left(m_{\mathrm{V}}=11.7228\right.$, van Leeuwen 2007) isolated WD (with a well known distance of 57.96 pc, Anderson \& Francis 2012) of the sample, is ideal for panchromatic calibration from the ultraviolet (UV) to the infrared (IR) wavelength range. However, due to its high $T_{\text {eff }}$ and relatively low $\log g$, radiative levitation competes against gravitational settling and holds trace elements in the photosphere and exhibits many weak metal lines (e.g. Barstow et al. 2003) in its observed UV spectrum.

A variety of previous spectral analyses of G191-B2B (Table 2) had shown that it is difficult to determine its $T_{\text {eff }}$ precisely. Barstow et al. (1998) found that the metal content in the photosphere has a strong impact on the determined $T_{\text {eff }}$. $T_{\text {eff }}=60920 \mathrm{~K}$ was found by the most recent analysis (Gianninas et al. 2011) who considered only $\mathrm{C}, \mathrm{N}$, and $\mathrm{O}$ (at solar abundances) in their models. The neglect of other metals calls for improved models with better metal opacities. The same may be true for HZ 43A even if metals are below the detection limit of the available spectra (Table 1).

Many abundance analyses were performed, most of them (e.g. Barstow et al. 2005), were based on previous $T_{\text {eff }}$ determinations from Balmer-line fits (cf. Table 2) and not from selfconsistent fits to models with varying metal abundances. Lanz et al. (1996) measured He, C, N, O, Si, Fe, and Ni abundances, Holberg et al. (2003) determined abundances of C, N, O, Al, Si,

\footnotetext{
2 http://www.esa.int/Our_Activities/Space_Science/ Gaia_overview
}

$\mathrm{Fe}$, and $\mathrm{Ni}$ and gave upper limits for $\mathrm{Mg}, \mathrm{Cr}, \mathrm{Mn}$, and $\mathrm{Co}$. The compiled abundances are listed in in Table 3.

Based on a grid of state-of-the-art line-blanketed NLTE model atmospheres that include opacities of all identified metals, we perform a detailed spectral analysis. We describe the available observations in Sect. 2, followed by a brief introduction to our model atmosphere code and the atomic data (Sect. 3). The spectral analysis is summarized in Sect. 4 and we end with our conclusions (Sect. 7).

\section{Observations}

\subsection{FUSE data}

G191-B2B was observed many times over the course of the FUSE mission in the wavelength range $910 \AA-1190 \AA$, both for calibration purposes and for studies of the interstellar medium. For the present study, only observations obtained in the first eight months of the mission through the LWRS spectrograph aperture were analyzed. This time period included the majority of the LWRS exposure time obtained during the mission, and had the secondary benefit that the detectors had not yet suffered much degradation from gain sag. The observation IDs of the datasets were: M1010201, M1010202, M1030602, P1041203, and S3070101.

Apart from a few quirks affecting the M1010201 and M1030602 observations, which were among the first obtained during the mission, the quality of the data is excellent. No $\mathrm{SiC}$ data were obtained in observations M1010201 or M1030602 as a result of channel mis-alignment. Otherwise: exposure-toexposure variations in flux were typically well under $1 \%$, indicating good channel alignment. The detector region used to record spectral image data for $\mathrm{LiF} 2 \mathrm{~b}$ was offset from the actual spectrum position in the M1010201 observation, so those spectra were discarded. The net exposure times were $33.3 \mathrm{ks}$ for the $\mathrm{SiC}$ channels, $36 \mathrm{ks}$ for LiF2b, and $40 \mathrm{ks}$ for LiF1 and LiF2a.

No special processing was applied to data from individual exposures. Raw data were processed with CalFUSE v3.2.3. Zero-point offsets in the wavelength scale were adjusted for each exposure by shifting each spectrum to coalign narrow interstellar absorption features. In order to assess the influence of geocoronal airglow emission, spectra obtained during orbital day and night were combined separately. All the observations were obtained in spectral image ("histogram") mode, so no information on photon arrival time was available within an exposure. However, the timeline table in the intermediate data files was examined for each exposure to determine the time spent in the "Day" and "Night" portions of the orbit. If the "Day" portion of such an exposure exceeded $15 \%$ of the total exposure duration, it was included with the other Day spectra. Because histogram mode exposures were short, most exposures were entirely Day or entirely Night.

The individual exposures from all five observations were then combined to form composite Day and Night spectra for each channel. The Day and Night spectra were then compared at the locations of all the known airglow emission lines. If the Day spectra showed any excess flux in comparison to the Night spectra at those locations, the corresponding pixels in the Day spectra were flagged as bad and were not included in subsequent processing. Significant airglow emission during orbital Day was seen for most observations at $\mathrm{H} \mathrm{I} \mathrm{Ly} \beta$ through Ly $\delta$, and $\mathrm{O}_{\text {I }} \lambda \lambda 988,1027,1028,1039 \AA$. Significant airglow was present during orbital night only at Ly $\beta$; this affects the 
interstellar-absorption profile but has no effect on our analysis of the photospheric spectrum.

The final step was to combine the spectra from the four instrument channels into a single composite spectrum. Because of residual distortions in the wavelength scale in each channel, additional shifts of localized regions of each spectrum were required to coalign the spectra; such shifts were typically only one or two pixels. Bad pixels resulting from detector defects were flagged at this point and excluded from further processing. Finally, the spectra were resampled onto a common wavelength scale and combined, weighting by signal to noise on a pixel-by-pixel basis.

The signal to noise of the final combined spectrum is limited by fixed-pattern noise in the detectors. The final spectrum has a minimum of roughly 20000 counts per $0.013 \AA$ pixel in the continuum, near the Lyman edge, and 60000-130000 counts per pixel long-ward of $1000 \AA$. The effects of fixed-pattern noise are minimized by the fact that the positions of the spectra on the detectors varied during each observation, and by the fact that nearly every wavelength bin was sampled by at least two different detectors.

\subsection{HST data}

As described in detail by Bohlin \& Gordon (in prep.), the HST/STIS low-dispersion flux calibration is derived from an ensemble match to the NLTE TLUSTY (version 203) model atmosphere SEDs for pure hydrogen (Hubeny \& Lanz 1995). The models are for G191-B2B, GD 71, and GD 153. Originally, HZ 43A was also used as a standard star but fell off the list of primary flux standards because of an $\mathrm{M}$ star companion that contaminates the STIS observations in the visible and IR (Bohlin et al. 2001).

For the STIS échelle modes, the flux calibration is based only on the TLUSTY model for G191-B2B. The échelle absolute fluxes are less precise than for low dispersion because of the single model for the reference fluxes, because of imprecision in the matching of the separate echelle orders, and because the plethora of weak lines at the shorter wavelengths are missing in the reference SED. However, the STIS echelle narrow metal line profiles are unaffected by uncertainties in the absolute fluxes.

For the highest STIS resolution of $\approx 3 \mathrm{~km} \mathrm{~s}^{-1}$, there are two modes, namely E140H and $\mathrm{E} 230 \mathrm{H}$, which require several central wavelength settings for complete wavelength coverage from 1145-3145 $\AA$. Because G191-B2B is the primary STIS échelle calibration star with repeated observations, $105 \mathrm{ob}-$ servations in the $0.2 \times 0.2$ aperture are available from the Mikulski Archive for Space Telescopes (MAST) ${ }^{3}$. Each spectrum is resampled to a wavelength grid with a sampling interval corresponding to a resolving power of $R=2.3 \times 10^{5}$ and co-added. The number of individual observations at each wavelength point ranges from 4-44, while the total exposure time ranges from 6400-64000 s at each point. The total counts in electrons at each point in the continuum are typically well above 1000 and range up to over 10000 from $1225-1400 \AA$, where the statistical uncertainty is sometimes better than $1 \%$. The highdispersion échelle spectrum is available from the CALSPEC ${ }^{4}$ database along with the STIS low-dispersion data.

\footnotetext{
3 http://archive.stsci.edu/

4 http://www.stsci.edu/hst/observatory/cdbs/calspec. html
}

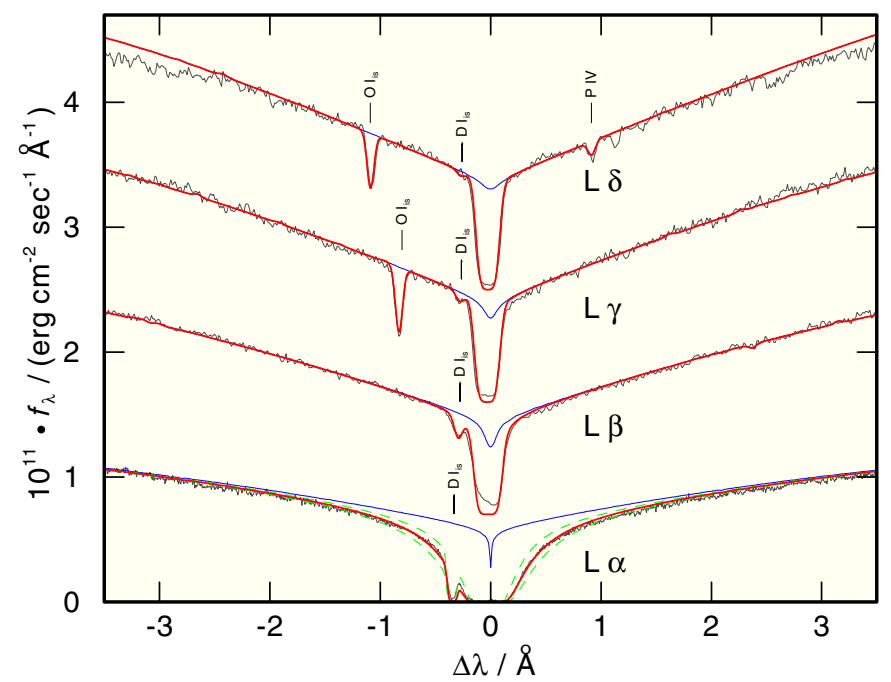

Fig. 2. Comparison of STIS and FUSE observations around H I L $\alpha-\delta$ with our final model. Thick (red in the online version) photospheric + ISM line absorption model with $N_{\mathrm{HI}}=2.2 \times 10^{18} \mathrm{~cm}^{-2}$; thin (blue) pure photospheric model; dashed (green, $\mathrm{L} \alpha$ only) $N_{\mathrm{HI}}=1.2 \times$ $10^{18} \mathrm{~cm}^{-2}, 3.2 \times 10^{18} \mathrm{~cm}^{-2}$. The locations of the D I blends are marked. $\mathrm{L} \beta-\delta$ are shifted in flux $\left(0.7,1.6,2.7 \times 10^{-11} \mathrm{erg} \mathrm{cm}^{-2} \mathrm{~s}^{-1} \AA^{-1}\right)$ for clarity. A reddening of $E_{B-V}=0.0005$ is applied following the law of Fitzpatrick (1999, with $\left.R_{\mathrm{V}}=3.1\right)$.

The photospheric radial velocity $v_{\text {rad }}=22.1 \pm 0.6 \mathrm{~km} \mathrm{~s}^{-1}$ measured by Holberg et al. (1994) matches well our STIS observation. We adopt this value for our analysis.

\subsection{Interstellar line absorption and reddening}

The interstellar neutral hydrogen density $N_{\mathrm{HI}}$ was determined from the comparison of our final model with the STIS and FUSE observations (Fig. 2). In all plots shown in this paper, we modeled the interstellar medium (ISM) line absorption (using Voigt line profiles) with WRPLOT ${ }^{5}$. The best match is found for $\log \left(N_{\mathrm{HI}} / \mathrm{cm}^{2}\right)=18.34_{-0.10}^{+0.08}$. The D I blends to H I L $\alpha-\delta$ are clearly visible and best reproduced at $\log \left(N_{\text {DI }} / \mathrm{cm}^{2}\right)=$ $13.54_{-0.06}^{+0.05}$, i.e. $D / H=1.59_{-0.65}^{+0.41} \times 10^{-5}$, Our values are in good agreement with those determined by Lemoine et al. (2002), $\log \left(N_{\mathrm{HI}} / \mathrm{cm}^{2}\right)=18.18 \pm 0.18$ and $D / H=1.66_{-0.6}^{+0.9} \times 10^{-5}$ (both with $2 \sigma$ errors).

Besides H I and D I, we identified interstellar lines of C II- IV, N I-II, O I, Al II, Si II-III, P I- II, S I-II, and Fe II in the FUSE and STIS spectra (Table 5). To identify pure photospheric lines that are contaminated by ISM lines, we modeled all of these and found that we need two distinct clouds with $v_{\text {rad }}=9 \pm 1 \mathrm{~km} \mathrm{~s}^{-1}$ and $v_{\text {rad }}=19 \pm 1 \mathrm{~km} \mathrm{~s}^{-1}$. This is well in agreement with the measurements of Sahu et al. $\left(8.6 \pm 1.7 \mathrm{~km} \mathrm{~s}^{-1}\right.$ and $19.3 \pm 2.5 \mathrm{~km} \mathrm{~s}^{-1}$ 1999), who assigned the latter value to the local interstellar cloud. Dickinson et al. (2012b) measured $8.5 \pm 0.18 \mathrm{~km} \mathrm{~s}^{-1}$ and $19.3 \pm 0.03 \mathrm{~km} \mathrm{~s}^{-1}$. They unambiguously detected that the first cloud is of circumstellar origin. An additional third cloud with intermediate velocity like assumed by Vidal-Madjar et al. $\left(1998, v_{\text {rad }}=8.2,13.2,20.3\left( \pm 0.8 \mathrm{~km} \mathrm{~s}^{-1}\right)\right.$ is not necessary for our modeling (Fig. 3, top).

Interestingly, we find additional weak absorptions of O I $\lambda 1302.163 \AA$ and $\mathrm{N}_{\mathrm{I}} \lambda 1199.550,1200.223,1200.710 \AA$ at $v_{\text {rad }}$ of $-26.3 \mathrm{~km} \mathrm{~s}^{-1}$ and $-26.1, \mathrm{~km} \mathrm{~s}^{-1}$, respectively. These

\footnotetext{
5 http://www.astro.physik.uni-potsdam.de/ htodt/ wrplot/index.html
} 


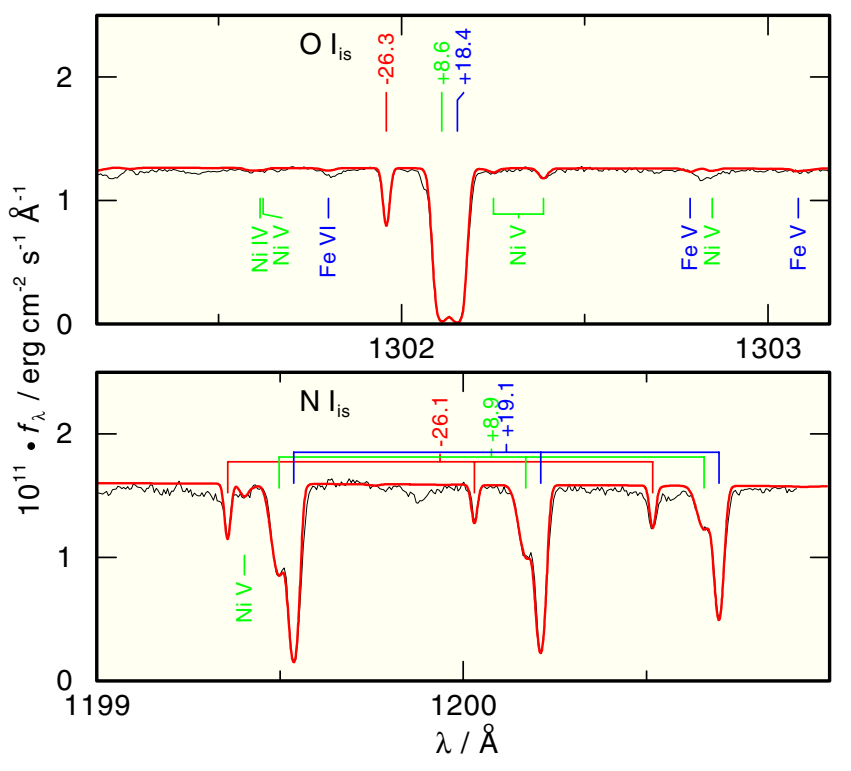

Fig. 3. STIS spectrum around the interstellar absorption lines O I $\lambda 1302.163 \AA$ (top) and N I $\lambda \lambda 1199.550,1200.223,1200.710 \AA$ (bottom) compared with the synthetic spectrum of our final model where the ISM lines are included. The labels give the radial velocities (in $\mathrm{km} \mathrm{s}^{-1}$ ) that are applied.

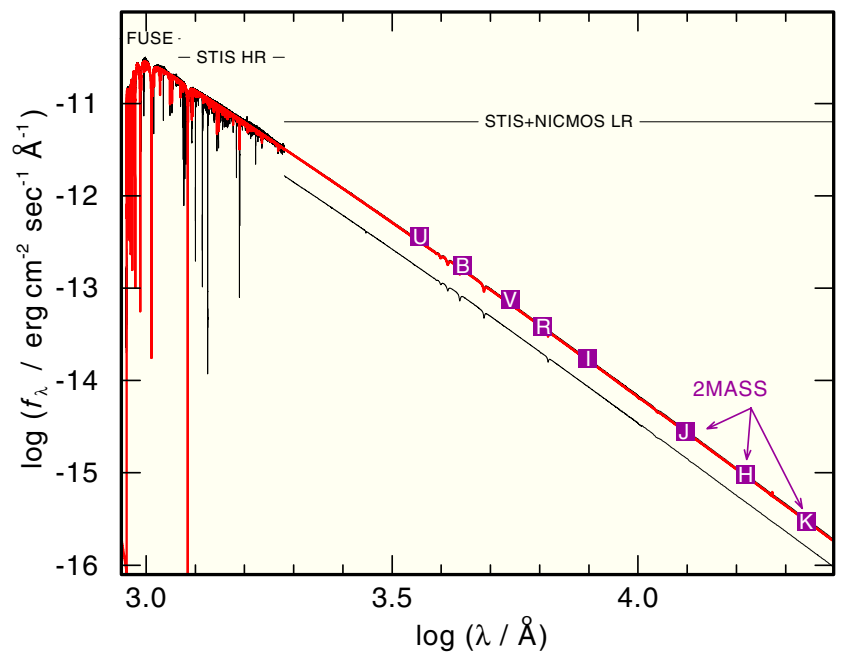

Fig. 4. Comparison of FUSE and HST (STIS and NICMOS) observations with our final model. A reddening of $E_{B-V}=0.0005$ is applied. The low-resolution (LR) STIS+NICMOS observation vanishes behind the model SED due to the line width. Therefore, we plotted the observed spectrum twice, one shifted by $\Delta \log f_{\lambda}=-0.2$ for clarity. $U, R, I$ (Landolt \& Uomoto 2007), $B, V$ (Høg et al. 2000), $J, H$, and $K$ (Cutri et al. 2003) fluxes (converted from brightnesses using values given by Heber et al. 2002) are shown for comparison.

velocities are reminiscent of the expansion velocity of a planetary nebula shell (e.g.. Kwok et al. 1978), that for a stellar mass of $M=0.555 M_{\odot}$ (Sect. 4.6) must have been ejected more than 500000 years ago (Renedo et al. 2010). Its recombined, neutral gas, however, is still in the line of sight.

From the low interstellar $N_{\mathrm{H}}$ density, we expect a low interstellar reddening. The Galactic reddening law of Groenewegen $\&$ Lamers (1989), $\log \left(N_{\mathrm{HI}} / E_{B-V}\right)=21.58 \pm 0.10$, predicts $0.0003 \lesssim E_{B-V} \lesssim 0.0007$. Figure 4 shows a comparison of observations and synthetic spectrum from the far UV (FUV) to the IR. We find $E_{B-V}=0.0005 \pm 0.0005$.

\section{Model atmospheres and atomic data}

Table 2 demonstrates clearly that a panchromatic analysis from the EUV to the optical is inevitable for accurate results on photospheric parameters. Moreover, NLTE modeling is mandatory to calculate a reliable synthetic spectrum.

Lanz et al. (1996) presented the first NLTE model (Table 2) that reproduced the observed spectrum from the EUV to the optical wavelength range. Barstow \& Hubeny (1998) introduced then a stratified $\mathrm{H}+\mathrm{He}$ envelope including heavier metals in their models to improve the match to the observed flux below the He II absorption threshold $(\lambda \lesssim 228 \AA$ ). Later analyses had shown that there is further evidence for a stratification in G191-B2B's photosphere. Vennes et al. (2000) closely examined Feige 24 that, compared with G191-B2B, has similar atmospheric parameters and an almost identical abundance pattern. They found that the $\mathrm{OIV} / \mathrm{OV}$ ionization equilibrium is overcorrected by -0.8 dex in their NLTE model. They concluded that this might reveal an inhomogeneous vertical stratification of oxygen in both stars. A later analysis of both stars (Vennes \& Lanz 2001) showed that the average heavy-metal abundance in Feige 24 is 0.17 dex larger compared to the cooler and, hence, older G191-B2B (same $\log g$ ). Thus, the abundance pattern is determined by the same processes in both stars and the authors assumed that selective radiative pressure and gravity are in diffusive equilibrium. This was proven by Dreizler \& Wolff (1999). They used self-consistent diffusion models (Table 2) that were able to reproduce the observed flux for $\lambda \lesssim 228 \AA$ without additional absorbers or mechanisms. However, problems remained with the fit to the UV lines.

Now, our strategy to proceed with the analysis is threefold. We start with chemically homogeneous models to find the model that reproduces best the continuum slope and the spectral lines from the FUV to the optical (Sect. 4). In an intermediate step, we will then apply the depth-dependent abundance profiles calculated by Dreizler \& Wolff (1999) to our final homogeneous model to investigate the impact of chemical stratification on the emergent spectrum (Sect. 4.3). In the last step, a diffusion model is calculated and compared with the homogeneous model (Sect. 4.4).

\section{Spectral analysis and results}

The metal-line blanketed NLTE model atmospheres for our analysis were calculated with the state-of-the-art Tübingen NLTE model-atmosphere package (TMAP 6 , Werner et al. 2003), which can consider opacities of all elements from $\mathrm{H}$ to $\mathrm{Ni}$ and beyond (Rauch 1997, 2003; Werner et al. 2012; Rauch et al. 2012). TMAP was successfully used for the spectral analysis of hot, compact stars (e.g. Rauch et al. 2007; Wassermann et al. 2010; Ziegler et al. 2012).

Our models assume plane-parallel geometry and are in hydrostatic and radiative equilibrium. Opacities of all species for which spectral lines are identified, namely $\mathrm{H}, \mathrm{He}, \mathrm{C}, \mathrm{N}, \mathrm{O}, \mathrm{Al}$, Si, P, S, Ca, Sc, Ti, V, Cr, Mn Fe, Co, Ni, Zn, Ge, and Sn, were considered in the model-atmosphere calculations. For all elements, we account for level dissolution (pressure ionization) following Hummer \& Mihalas (1988) and Hubeny et al. (1994). Figure 5 demonstrates that our H I model ion (Table 4) includes all levels that are relevant in the line-forming region $-4.5<$ $\log \left[\mathrm{m} /\left(\mathrm{g} / \mathrm{cm}^{2}\right)\right]$. All model atmospheres presented here cover

6 http://astro.uni-tuebingen.de/ TMAP 
T. Rauch et al.: TheoSSA: Establishing a database of synthetic stellar flux standards

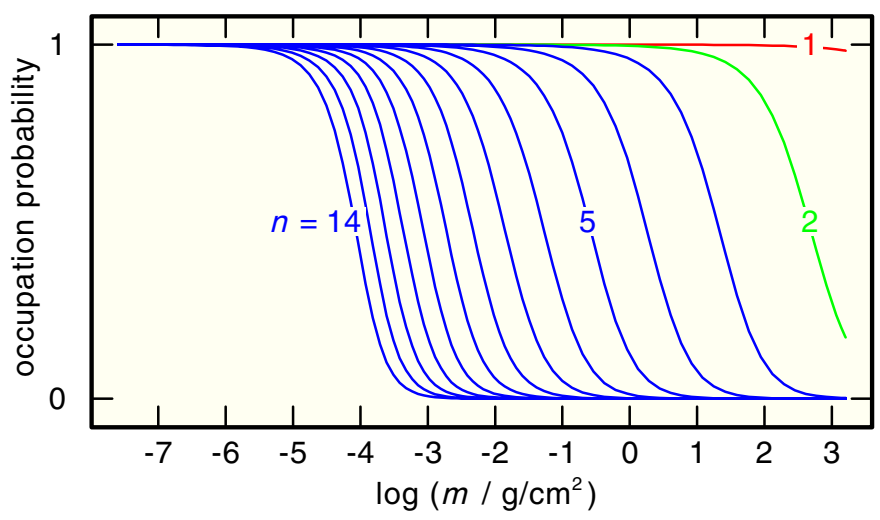

Fig. 5. Occupation probabilities of the H I levels with principal quantum numbers $n=1-14$ in our final model.

column densities $m$ of $-7.6<\log m<3.2$ (cf. Beuermann et al. 2006) represented by 90 depth points.

The model-atoms and respective absorption cross-sections for $\mathrm{Ca}-\mathrm{Ni}$ were calculated via the recently registered $\mathrm{VO}$ service TIRO $^{7}$ that uses Kurucz's atomic data ${ }^{8}$ and line lists (Kurucz 1991, 2009, 2011).

$\mathrm{Ca}, \mathrm{Sc}, \mathrm{Ti}, \mathrm{V}, \mathrm{Cr}, \mathrm{Mn}$, and Co lines are not identified. These were merged into a generic model atom (Rauch \& Deetjen 2003) with fixed solar abundance ratios. Then, we performed test calculations and adjusted the abundance to a value $\left(1.78 \times 10^{-6}\right.$ by mass, the solar value is $9.93 \times 10^{-5}$ ) where all of its lines just fade in the noise of the observed spectra. All other model atoms were constructed from data retrieved from the public Tübingen model-atom database TMAD ${ }^{9}$.

In total, we considered 1038 atomic levels in NLTE combined with 4646 line transitions (for the number of individual iron-group lines, see Table 4) in the model-atmosphere calculations with 53203 frequency points within $1 \times 10^{12} \mathrm{~Hz} \leq v \leq$ $3 \times 10^{17} \mathrm{~Hz}$. For the emergent spectra $(100 \AA \leq \lambda \leq 400000 \AA$, 686196 wavelength points), we account for fine-structure splitting and used 1585 NLTE levels and 9721 respective line transitions. The model-atom statistics are summarized in Table 4. Figure 6 shows the ionization fractions of all elements in our final model. It may be interesting to note that a single model atmosphere needs about one week to converge, i.e. the absolute values of all relative corrections are below $10^{-4}$, on a $64 \mathrm{bit}, 2.66 \mathrm{GHz}$ compute core with 8 GB memory.

For the calculation of synthetic Hi line profiles, we use Stark line-broadening tables provided by Tremblay \& Bergeron (2009). For those lines, where no broadening tables are available, TMAP uses an approximate formula, as described in Ziegler et al. (2012, Eqs. (1)-(5)).

We started with a model with $T_{\text {eff }}=60920 \mathrm{~K}$ and $\log g=7.55$ (the values of Gianninas et al. 2011) and the element abundances from Table 3. Next, we adjusted these abundances to best reproduce the respective spectral lines. We then calculated an extended grid of 234 model atmospheres $\left(48000 \mathrm{~K} \leq T_{\text {eff }} \leq\right.$ $68000 \mathrm{~K}$ in steps of $\leq 1000 \mathrm{~K}$ and $7.35 \leq \log g \leq 7.75$ in steps of 0.05 (some of the hotter models are calculated only for $\log g \leq 7.60$ ). For this grid, we extensively used compute resources of the bwGRiD ${ }^{10}$ in addition. Although this highly speeded up the model-grid calculation, the wide parameter range

\footnotetext{
7 http://astro.uni-tuebingen.de/ TIR0

8 http://kurucz.harvard.edu/atoms.html

9 http://astro.uni-tuebingen.de/ TMAD

${ }^{10}$ http://www.bw-grid.de/en/the-bwgrid/
}

and the large number (15) of parameters to adjust simultaneously did not allow us to take a statistical approach in the spectral analysis ( $\chi^{2}$ method like e.g. in Gianninas et al. 2011) on a reasonable time scale. We therefore need to rely upon our " $\chi$-by-eye" methods. All SEDs that were calculated for this analysis are available via TheoSSA ${ }^{11}$.

In a first analysis step, we will determine $\log g$ based on UV and optical observations. Then, we will determine $T_{\text {eff }}$ precisely based on ionization equilibria of the metals which are sensitive indicators. Subsequently, we will adjust the abundances again and verify our $T_{\text {eff }}$ and $\log g$ results.

\subsection{Surface gravity and effective temperature}

The dependency of the synthetic flux level on $T_{\text {eff }}$ and $\log g$ for fixed abundances is demonstrated in Fig. 7, where we compare the observed and synthetic fluxes in the FUV. In the top panel, it is obvious that at a constant $T_{\text {eff }}=60920 \mathrm{~K}$, a $\log g$ higher by 0.2 dex than $\log g=7.55$ measured by Gianninas et al. (2011) is necessary to reproduce the Lyman-line decrement. For a fixed $\log g=7.55$ (middle panel), a lower $T_{\text {eff }}\left(\Delta T_{\text {eff }}=6000 \mathrm{~K}\right)$ improves the agreement between model and observation. The bottom panel shows that at values within the (statistical) error ranges from the H I Balmer-line analysis, $T_{\text {eff }}=60000 \mathrm{~K}$ and $\log g=7.60$ (cf. Table 2 Gianninas et al. 2011), a good agreement for both, line profiles and decrement, is achieved.

This was not expected from the outset although Barstow et al. (1998) found a relatively good agreement of $T_{\text {eff }}$ and $\log g$ from H I Lyman and Balmer lines in the heavy-metal rich models (Table 2). The later analysis by Barstow et al. (2001, Table 2) shows strong deviations in $\log g$ between optical and FUV analyses. Figure 8 shows a comparison of synthetic H I Balmer line profiles with optical observations. The deviation between the $T_{\text {eff }}=60920 \mathrm{~K} / \log g=7.55$ and the $60000 / 7.60$ ones is minor.

In addition to the low-resolution $(R \approx 500)$ optical spectrum, medium-resolution $(R \approx 6000)$ observations of $\mathrm{H} \alpha$ and $\mathrm{H} \beta$ are shown is Fig. 9. The agreement among the STIS low and medium resolution is excellent. While the model absorption is a bit weak as shown in the lower plot of Fig. 9, the central NLTE emission reversal agrees well with the observations.

Although we cannot reproduce $\mathrm{H} \alpha$ and $\mathrm{H} \beta$ in detail in the medium-resolution spectrum, this has no significant influence on our determination of $T_{\text {eff }}$ and $\log g$ because the higher members of the Hi Balmer series form much deeper in the atmosphere where the influence of metal opacities is less important (cf. Napiwotzki \& Rauch 1994). We adopt $\log g=7.60$.

In the next step of this analysis, we evaluate ionization equilibria of metals that exhibit lines of successive ionization stages. Figures 10-12 show some strategic lines for this $T_{\text {eff }}$ determination. In total, we can use eight elements and lines of C III- IV, N III- V, Si III- IV, P IV-V, S IV-V, Fe IV-VI, Ni IV-VI, and Ge IV-V. $T_{\text {eff }}=60000 \pm 2000 \mathrm{~K}$ reproduces well all these equilibria simultaneously. For our further analysis, we adopt $T_{\text {eff }}=60000 \mathrm{~K}$.

\subsection{Photospheric abundances}

In the following, we use logarithmic mass fractions for all abundance values, if not otherwise mentioned. Previously determined abundances and respective references are summarized in Table 3. In the following, we will briefly mention the strategic lines for

${ }^{11}$ http://dc.g-vo.org/theossa 


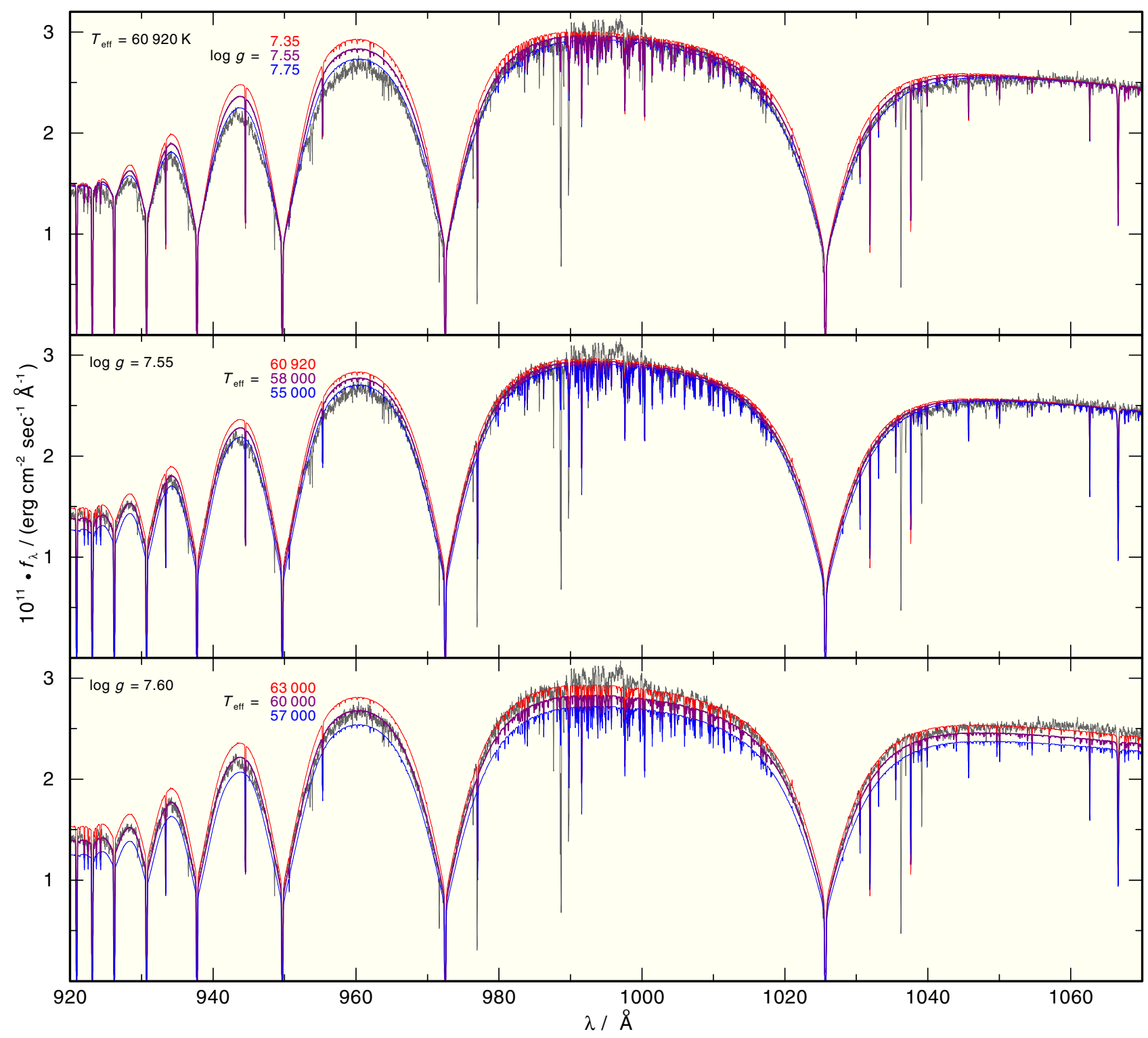

Fig. 7. Section of the FUSE observation compared with our model fluxes with different $T_{\text {eff }}$ and $\log g$. In the top and middle panels, the synthetic fluxes are normalized to the observed flux at $1000 \AA$ and in the bottom panel to the observed $K$ magnitude (see Fig. 4). $E_{B-V}$ and $N_{\mathrm{HI}}$ are applied using our results from Sect. 2.3.

the abundance determinations and note abnormalities for an element selection only. Most of the identified metals exhibit lines of at least two subsequent ionization stages and some of these lines were already used for the determination of $T_{\text {eff }}$ (Sect. 4.1). The abundances were then adjusted to achieve best line fits. Two large plots (Figs. A.1, A.2, German DIN A0 size) are provided in the online material that show a comparison of our final model with the observation in the FUSE and STIS wavelength ranges (in total 911-1750 ̊). They include all line identifications (FUSE/STIS wavelength range), e.g. 2/421 Fe IV, 144/815 Fe V, 1/52 Fe VI, 1/236 Ni IV, 13/690 Ni V, and 9/43 Ni VI lines. These numbers are much higher than those of Preval et al. (2013, $106 \mathrm{Fe} \mathrm{V}$ and $44 \mathrm{Ni} \mathrm{V}$ lines in the STIS wavelength range). The recent work of Berengut et al. (2013) to employ G191-B2B as a stellar laboratory to determine the fine-structure constant is based on the latter list and may, thus, not fully exploit capacity of all the available STIS spectra of G191-B2B.

Our line identifications are also summarized in Table 5, whereas Table 6 gives a list of the strongest unidentified lines.

\subsubsection{Helium}

The first analyses revealed only upper limits for the He abundance, e.g. He < -3.1 and <4.1 (Vennes et al. 1996; Gunderson et al. 2001, respectively). Cruddace et al. (2002) determined $\mathrm{He}=-4.2 \pm 0.1$ using high-resolution EUV spectroscopy. An attempt to identify and measure He II Lyman lines $\left(n-n^{\prime}=1-4\right.$, $1-5)$ with J-PEX ${ }^{12}$ (Barstow et al. 2005) was not successful. Our models show that He II $\lambda 1640 \AA$ (2-3) should be clearly visible at $\mathrm{He}=-3.7$ and -4.2 and disappears in the noise of the observation only at about $\mathrm{He}<-4.7$ (Fig. 13). We adopt this upper-limit value for our models.

\subsubsection{Carbon, nitrogen, and oxygen}

C III and C IV lines are visible in the observation. C III $\lambda 977.02 \AA$ and $C_{\text {IV }} \lambda \lambda 1548.20,1550.77 \AA$ have strong ISM blends. In case

12 Joint Astrophysical Plasmadynamic Experiment 
T. Rauch et al.: TheoSSA: Establishing a database of synthetic stellar flux standards

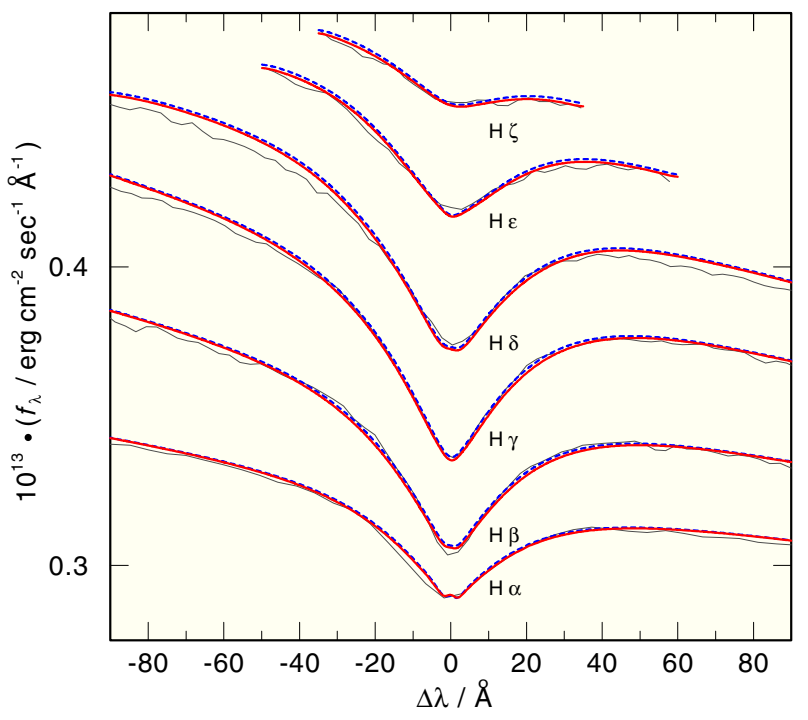

Fig. 8. Synthetic H I Balmer-line profiles compared with the STIS LR observation. The observation is shifted by factors of $0.921,0.323$, $0.232,0.202,0.193$, and 0.188 for $\mathrm{H} \alpha-\mathrm{H} \zeta$, respectively, to fit into this plot. Red, full line: $T_{\text {eff }}=60000 \mathrm{~K}$ and $\log g=7.60$; blue, dashed: $T_{\text {eff }}=60920 \mathrm{~K}$ and $\log g=7.55$.

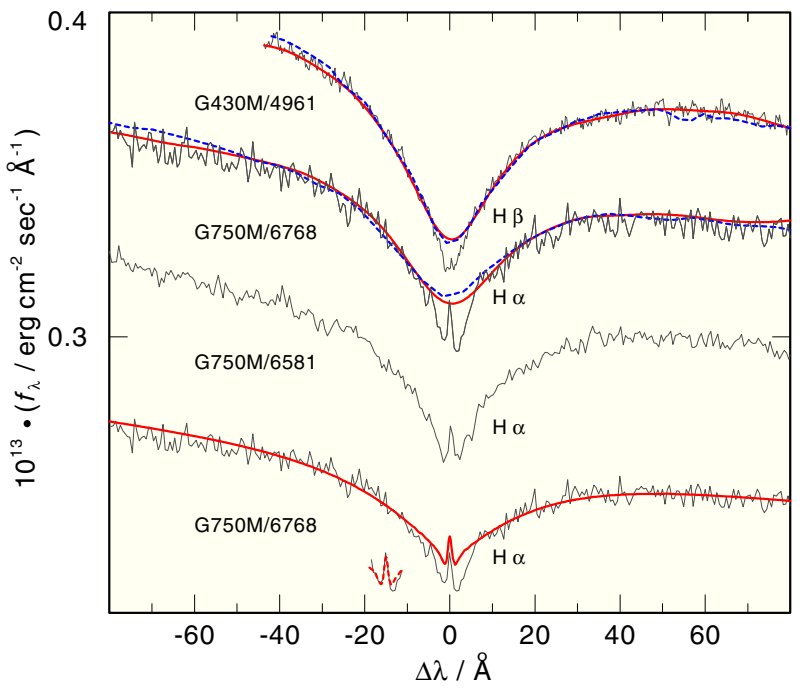

Fig. 9. STIS $\mathrm{H} \alpha$ and $\mathrm{H} \beta$ low-resolution (dashed, blue) and mediumresolution (gray lines) observations (labeled with medium-resolution grating/central wavelength in $\AA$ ). Because of uncertainty in the flux calibration, the medium-resolution data are normalized to the lowresolution flux. The flux around $\mathrm{H} \beta$ in the top plot is multiplied by a factor of 0.35 . The red lines in the upper two plots are the mediumresolution $(R \approx 6000)$ spectra degraded to the low resolution $(R \approx 500)$ and agree with the low resolution (blue dash) within the uncertainty of the $R=500$ resolution. The lower two plots are shifted down by 0.035 and $0.07 \times 10^{-13}$ flux units, respectively. In the lowest plot, the model is overplotted in red after smoothing to the medium resolution. While the model $\mathrm{H} \alpha$ absorption is somewhat too weak, the central emission agrees with the observation within the uncertainty of the resolution (insert).

of the latter, the photospheric component can be separated and modeled (Fig. 10). At $C=-5.15$, lines of both ions are well reproduced.

$\mathrm{N}$ III- $\mathrm{V}$ lines are found in the observation, they are all well matched at $N=-5.58$ (Fig. 10).

Vennes et al. (2000) encountered deviations between oxygen abundances determined from O IV and OV lines in an
Table 6. Wavelengths (in $\AA$ ) of unidentified strong $\left(W_{\lambda}>10 \mathrm{~m} \AA\right.$ ), likely photospheric lines in the FUSE and STIS observations.

\begin{tabular}{lllll}
\hline \hline FUSE & & & & \\
989.11 & 1029.44 & 1133.04 & 1142.91 & \\
\hline STIS & & & & \\
1157.60 & 1173.29 & 1183.82 & 1201.51 & 1283.52 \\
1158.08 & 1174.34 & 1186.08 & 1201.81 & 1306.04 \\
1158.76 & 1176.11 & 1186.27 & 1202.43 & 1331.19 \\
1165.31 & 1176.52 & 1186.59 & 1204.47 & 1385.32 \\
1166.80 & 1176.64 & 1187.70 & 1227.55 & 1389.89 \\
1171.12 & 1176.87 & 1190.15 & 1253.67 & 1398.21 \\
1171.19 & 1176.98 & 1192.01 & 1253.87 & 1411.46 \\
1171.45 & 1177.06 & 1194.16 & 1258.81 & 1516.68 \\
1172.18 & 1178.68 & 1198.15 & 1260.48 & 1520.64 \\
1172.34 & 1182.00 & 1201.30 & 1272.98 & 1525.32 \\
1173.22 & 1183.37 & 1201.47 & 1281.37 & 1538.94 \\
\hline
\end{tabular}

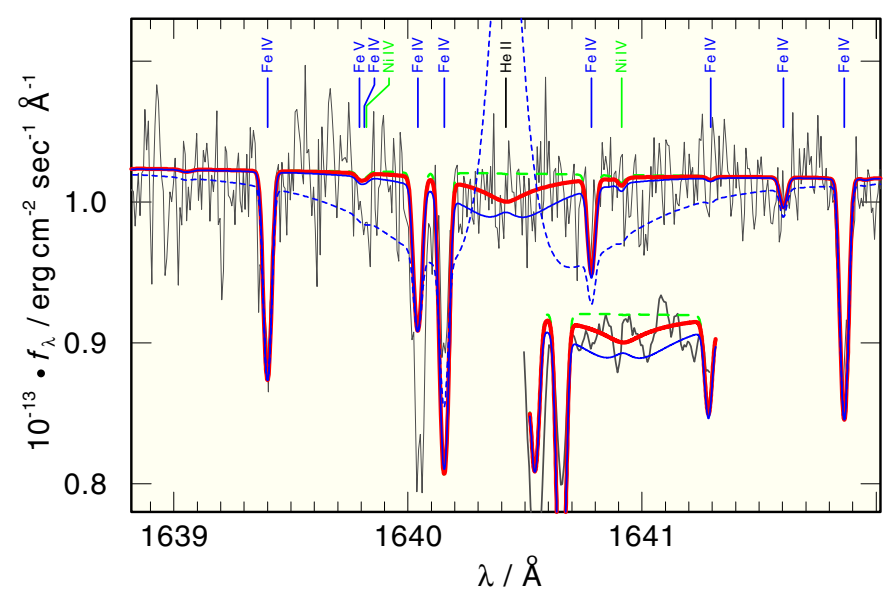

Fig. 13. Synthetic spectrum around He II $\lambda 1640.42 \AA$ compared with the STIS observation. He abundances are green, dashed: -10.0 , red, thick: -4.7 , blue, thin: -4.2 , blue, dashed: -3.1 . The insert shows the region $\Delta \lambda= \pm 0.4 \AA$ around the He II line. For comparison, the observation was smoothed with a low-pass filter (Savitzky \& Golay 1964, $n=15, m=4)$.

analysis of Feige 24. The $\mathrm{OV}$ abundance was 0.5 dex higher in their LTE model approach. In their NLTE models, they found that the O IV / O V ionization equilibrium was overcorrected by -0.8 dex. They suggested an inhomogeneous stratification of $\mathrm{O}$ in the atmosphere. Vennes \& Lanz (2001) discovered that a similar problem exists in G191-B2B, with an overcorrection of $-0.6 \mathrm{dex}$. Consequently they assumed that in both stars, the interplay between selective radiation pressure and gravity in diffusive equilibrium are the key processes for this phenomenon. Figure 14 shows the same deviation in our models. While O IV $\lambda \lambda 1338.615,1342.990,1343.526 \AA$ are well fitted at $O=-4.72, \mathrm{O} \vee \lambda 1371.296 \AA$ is apparently much stronger than observed. It is matched with an $\mathrm{O}$ abundance that is reduced by -0.4 dex.

In the FUSE observation, only the short wavelength component of the OVI $\lambda \lambda 1031.912,1037.614 \AA$ resonance doublet is detectable. The unexpected weakness of this doublet was already reported by Oegerle et al. (2005). Dickinson et al. (2012b) verified that it stems from the photosphere. The O VI resonance doublet in our models is even stronger, compared to $\mathrm{O} I \mathrm{~V}$ and $\mathrm{OV}$ lines, requiring a reduction of the $\mathrm{O}$ abundance by about -1.5 dex (Fig. 14). Dickinson et al. (2012a) encountered a similar problem with enigmatically deep line profiles of 


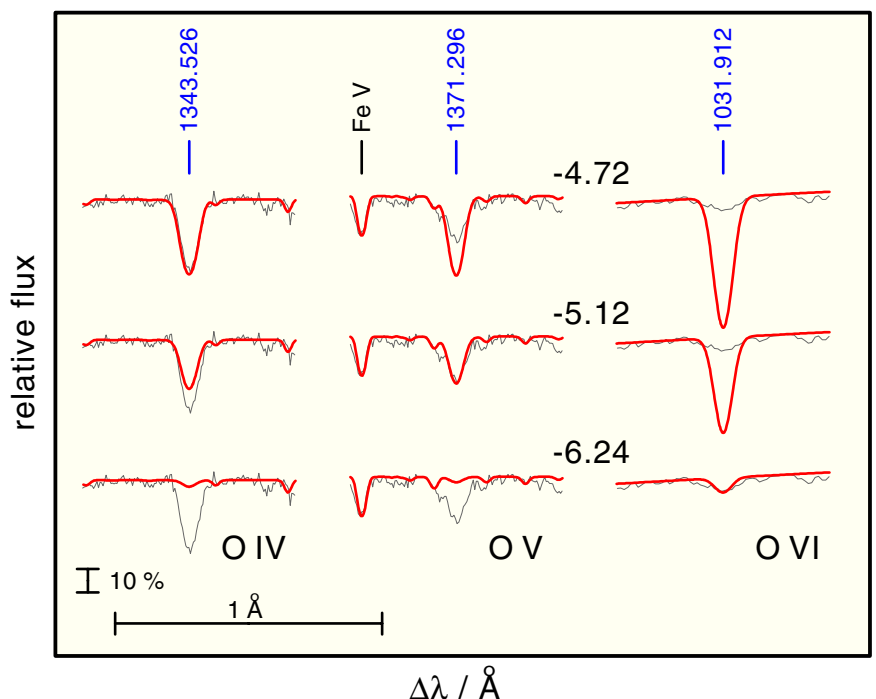

Fig. 14. Most prominent O IV, O v, and O VI lines in the STIS and FUSE observations compared with our theoretical line profiles calculated with three $\mathrm{O}$ abundances $(O=-4.72,-5.12,-6.24$, from top to bottom).

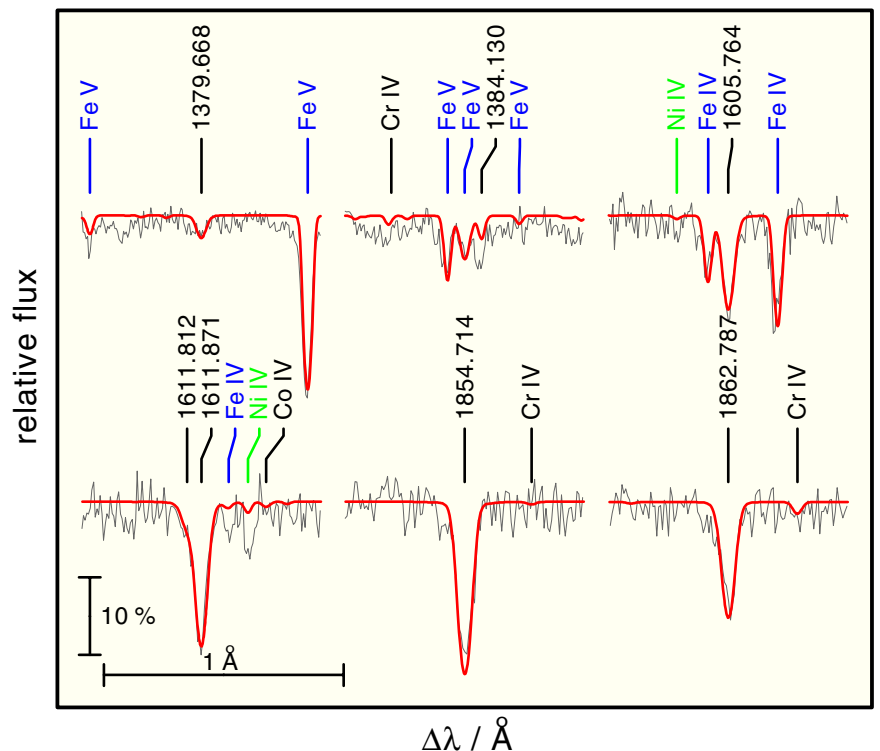

Fig. 15. Theoretical Al III line profiles calculated from our final model compared with the STIS observation.

the $\mathrm{N} v$ resonance doublet in their models. We revisit the problem with the oxygen abundances derived from different ionization stages in Sects. 4.3 and 4.4 in detail.

\subsubsection{Aluminum, silicon, phosphorus, and sulfur}

Holberg et al. (1998) identified the Al III $\lambda \lambda$ 1854.72, $1862.79 \AA$ resonance doublet in the IUE NEWSIPS SWP Echelle Data Set ${ }^{13}$, and Holberg et al. (2003) measured Al $=-5.08$. We could newly identify some other Al III lines. We derive $\mathrm{Al}=-4.95$, well in agreement with the Holberg et al. (2003) value (Fig. 15).

Si III- IV, P IV-V, and S V-VI lines are identified. We determine $\mathrm{Si}=-4.30, \mathrm{P}=-5.81$, and $\mathrm{S}=-5.24$ (Fig. 10).

\footnotetext{
${ }^{13}$ http://vega.lpl.arizona.edu/newsips/
}

\subsubsection{Iron-group elements}

Many hundreds of lines of Fe IV-VI and Ni IV-vi are identified (Table 5). They are best reproduced at $\mathrm{Fe}=-3.30$ and $\mathrm{Ni}=-4.45$. Note that the $\mathrm{Ni} / \mathrm{Fe}$ abundance ratio is about $25 \%$ higher than the solar ratio. Some of these lines are shown in various figures in this paper, please have a look at the two large online figures that show the complete FUSE and STIS wavelength ranges. An animation of STIS wavelength range can been seen at http://astro.uni-tuebingen.de/ rauch/AQ_E140H_SW.gif as well.

In Fig. 15 three lines of Cr IV and one of Co IV are visible in the synthetic spectrum of our final model. These are weak and comparable to the noise of the observation. Although one may be tempted to believe the presence of Cr IV $\lambda 1863.075 \AA$, we take this as a hint that a $\log$ mass fraction of -5.75 for the generic model atom is reasonable and adopt this as an upper limit for our analysis. This is, within the error limits, in agreement with the upper limits for $\mathrm{Cr}, \mathrm{Mn}$, and $\mathrm{Co}$ of about -6.2 that were found by Holberg et al. (2003).

Preval et al. (2013) suggested that the unidentified line at $1272.98 \AA$ is a V IV line. Since many other V IV lines with much stronger $\log g f$ values $(g$ is the statistical weight of the lower atomic level and $f$ is the oscillator strength of the line transition) from Kurucz's POS line lists (with good wavelengths) are not present in the spectrum, e.g. V IV $\lambda \lambda 1355.127,1419.577,1426.647 \AA$ (all more than ten times higher $\log g f$ ) therefore this identification appears to be very unlikely.

We mention here that we find deviations between Kurucz's POS wavelengths and the observation of up to $0.05 \AA$. In addition, Fig. 13 shows that the strengths of Fe IV $\lambda 1640.042 \AA$ and Fe IV $\lambda 1640.155 \AA$ in the model are the opposite way around in the observation.

Figure 16 shows a comparison of models (calculated with Kurucz's POS lines) in the FUSE and STIS wavelength ranges where in each case the abundance of an individual element $X$ in the construction of the generic ( $\mathrm{Ca}, \mathrm{Sc}, \mathrm{Ti}, \mathrm{V}, \mathrm{Cr}, \mathrm{Mn}, \mathrm{Co})$ model atom is increased by a factor of ten. Values higher that 1 in the flux ratio indicate stronger lines of element $X$.

E.g. the case of $\mathrm{Ti}$, two lines are much stronger than all others, Ti IV $\lambda \lambda 1451.739,1467.343 \AA$. They are not identified in the observation but at the ten times increased abundance they are clearly visible in the model. The same is valid for $\mathrm{Cr}$, where Cr IV $\lambda \lambda 1332.415 \AA$ and $\operatorname{Cr}$ vi $\lambda \lambda 1417.660 \AA$ are the strongest lines in our models (Fig. 16), and for Mn and Co as well. This allows us to establish upper abundance limits of about $10 \%$ solar for Ti, Cr, Mn, and Co (cf. the beginning of Sect. 4).

\subsubsection{Zinc, germanium, and tin}

21 Zn IV lines are newly identified in the STIS observation. These are almost all that are listed in the NIST ${ }^{14}$ database with relative intensities higher than 100. Since no individual calculations for Zn IV transition probabilities are available, we adapted those of the isoelectronic Ge VI (Rauch et al. 2012). In Fig. 17, we show nine of them with NIST relative intensities of 200. All their theoretical line profiles are reproduced at $Z n=-4.89$.

For Ge, we used the same model atom as Rauch et al. (2012) and determined $\mathrm{Ge}=-5.49$ (Fig. 10).

\footnotetext{
${ }^{14}$ http://physics.nist.gov/PhysRefData/ASD/lines_form. html
} 
T. Rauch et al.: TheoSSA: Establishing a database of synthetic stellar flux standards

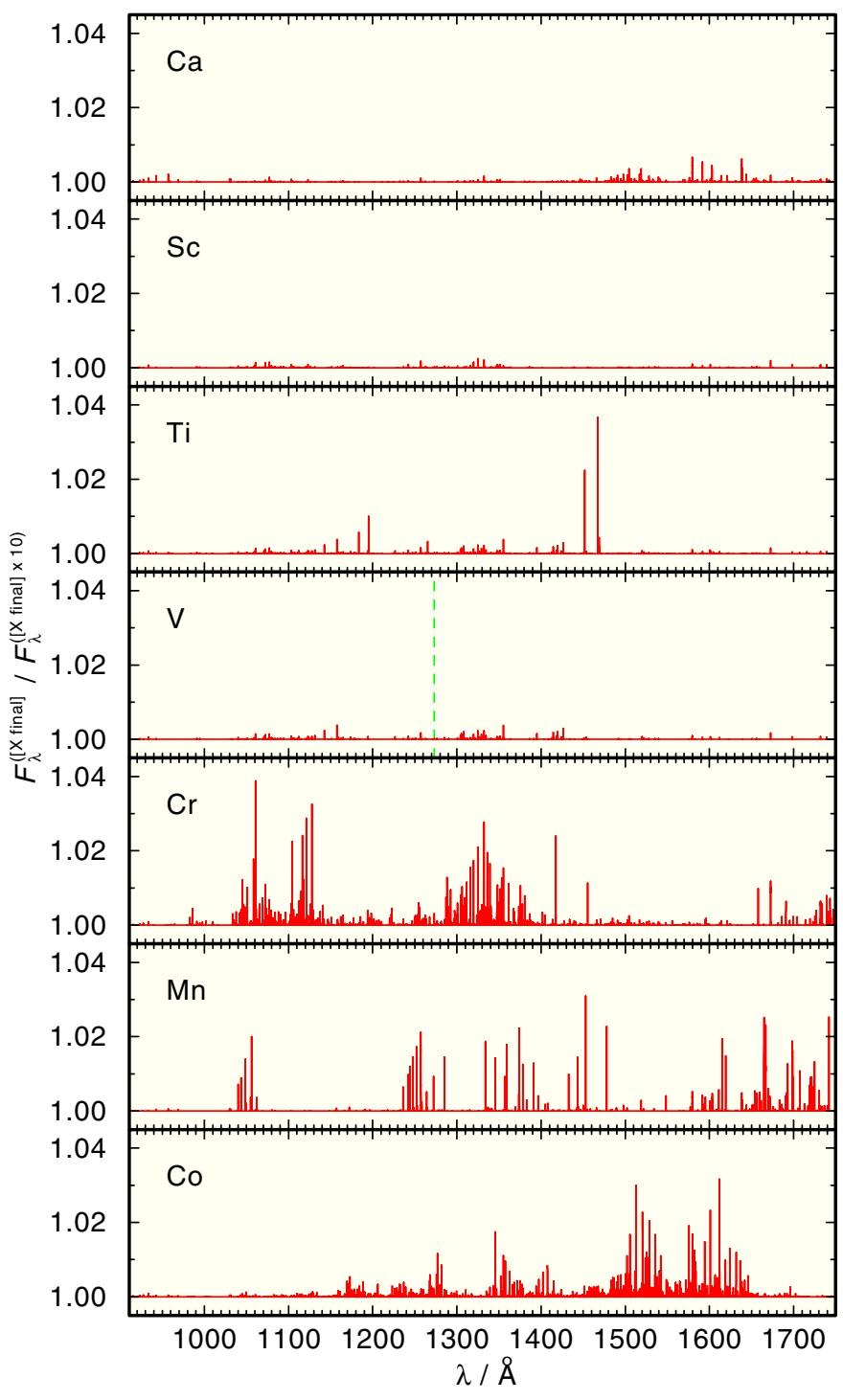

Fig. 16. Flux ratio of our final model and a model with ten times increased abundance of element $X$ (Ca to $\mathrm{Co}$, from top to bottom). The location of V IV $\lambda 1272.98 \AA$ is marked.

We constructed a relatively small $\mathrm{Sn}$ model atom. The only lines for which reliable oscillator strengths are available are the Sn III and Sn IV resonance lines (Morton 2000). For all other allowed transitions, we follow Werner et al. (2012) and set $f=1$. We used the Sn IV $\lambda 1314.537 \AA$ resonance line, like Vennes et al. (2005), to measure the abundance of $\mathrm{Sn}=-6.45$.

\subsubsection{Summary of results with chemically homogeneous models}

We can reproduce the entire ultraviolet spectrum of G191-B2B with our chemically homogeneous NLTE models, with the exception of the OIV/VI lines which are obviously affected by $\mathrm{O}$ stratification effects. Current diffusion models yield poor fits to the metal lines (Dreizler \& Wolff 1999). $T_{\text {eff }}=60000 \pm 2000 \mathrm{~K}$ and $\log g=7.60 \pm 0.05$ were determined within small error limits. They are in agreement with Gianninas et al. $\left(2011, T_{\text {eff }}=60920 \pm 993 \mathrm{~K}, \log g=7.55 \pm 0.05\right)$. We do not encounter problems in modeling Hi Lyman and Balmer lines simultaneously with the same $T_{\text {eff }}$ and $\log g$ like found by Barstow et al. (2001, see Table 2).
We can determine all abundances with error limits of 0.2 dex. In case of $\mathrm{Zn}$, where we adopt Ge VI $f$-values, we estimate that the error is 0.3 dex. Our $\mathrm{C}, \mathrm{N}, \mathrm{O}, \mathrm{Al}, \mathrm{Si}, \mathrm{Fe}$, and $\mathrm{Ni}$ abundances (Fig. 18) agree, within error limits, with those of Vennes et al. (1996); Holberg et al. (2003); Vennes et al. (2005). Our values are in general slightly higher. One reason may be the about $6000 \mathrm{~K}$ higher $T_{\text {eff }}$ of our final model. The stellar parameters are summarized in Table 8.

The abundances of all elements but Fe predicted by Chayer et al. (1995) for a DA-type WD differ strongly from those that we determined (Fig. 18).

\subsection{Test of the diffusion impact}

In a first step, we simply applied the abundance profiles provided by Dreizler \& Wolff (1999) to the occupation numbers of He, C, $\mathrm{N}, \mathrm{O}, \mathrm{Si}$, and $\mathrm{Ni}$ in our final model. Figure 19 (top panel) shows that this gives a good agreement with $\mathrm{O} v$ while $\mathrm{O}$ IV is now too weak. The O VI lines appears even stronger, strengthening the discrepancy. Since the atmospheric structure was kept fixed in this test, we expected that, if at all, only a self-consistent diffusion model is able to reproduce the observed OIV-VI lines simultaneously.

\subsection{A self-consistent diffusion model}

We used the NGRT ${ }^{15}$ code (Dreizler \& Wolff 1999; Schuh et al. 2002) to calculate diffusion models with the same element composition and model atoms like our homogeneous TMAP models. The first model shows a strongly increased abundance of the generic model atom that combines $\mathrm{Ca}, \mathrm{Sc}, \mathrm{Ti}, \mathrm{V}, \mathrm{Cr}, \mathrm{Mn}$, and Co (Sect. 4) and, hence, much too strong lines of the considered elements. The reason is that the IrOnIc code (Rauch \& Deetjen 2003) calculates a mean atomic weight for the generic atom following

$A_{\mathrm{IG}}=\frac{\sum_{i=1}^{n} r_{i} \cdot A_{i}}{\sum_{i=1}^{n} r_{i}}$

where $r_{\mathrm{i}}$ is the relative mass-fraction (with respect to $r_{1}=1$ ) and $A_{\mathrm{i}}$ the atomic weight of element $i$. The artificially increased number of lines of a single generic element strongly increases its radiative levitation. Flux blocking by the generic element then leads to stronger gravitational settling of other elements, e.g. Sn had an abundance below $10^{-17}$ throughout the model atmosphere. The other elements showed abundances that were partly more than one dex below those of our homogeneous model. Since we did not want to neglect all opacities of the generic atom, we changed its atomic weight to

$A_{\mathrm{IG}}=\sum_{i=1}^{n} r_{i} \cdot A_{i}$.

Now, the stratified NGRT models yields depth dependent abundances (Fig. 20) that are closer to those of our homogeneous model, especially $\mathrm{Sn}$ appears at a realistic value. In case of $\mathrm{He}$, $\mathrm{C}, \mathrm{N}, \mathrm{O}, \mathrm{Si}$, and $\mathrm{Ni}$ the abundance profiles are similar to those of (Dreizler \& Wolff 1999). The changed atmospheric structure is shown in Fig. 21. It is interesting to note that most of the lines and all continua are formed at $\log m \gtrsim-3$ (Fig. 22) while deviations in the temperature structure are noticeable only outside of this region. The resulting spectrum (Fig. 23) of the

${ }^{15}$ New generation radiative transport. 


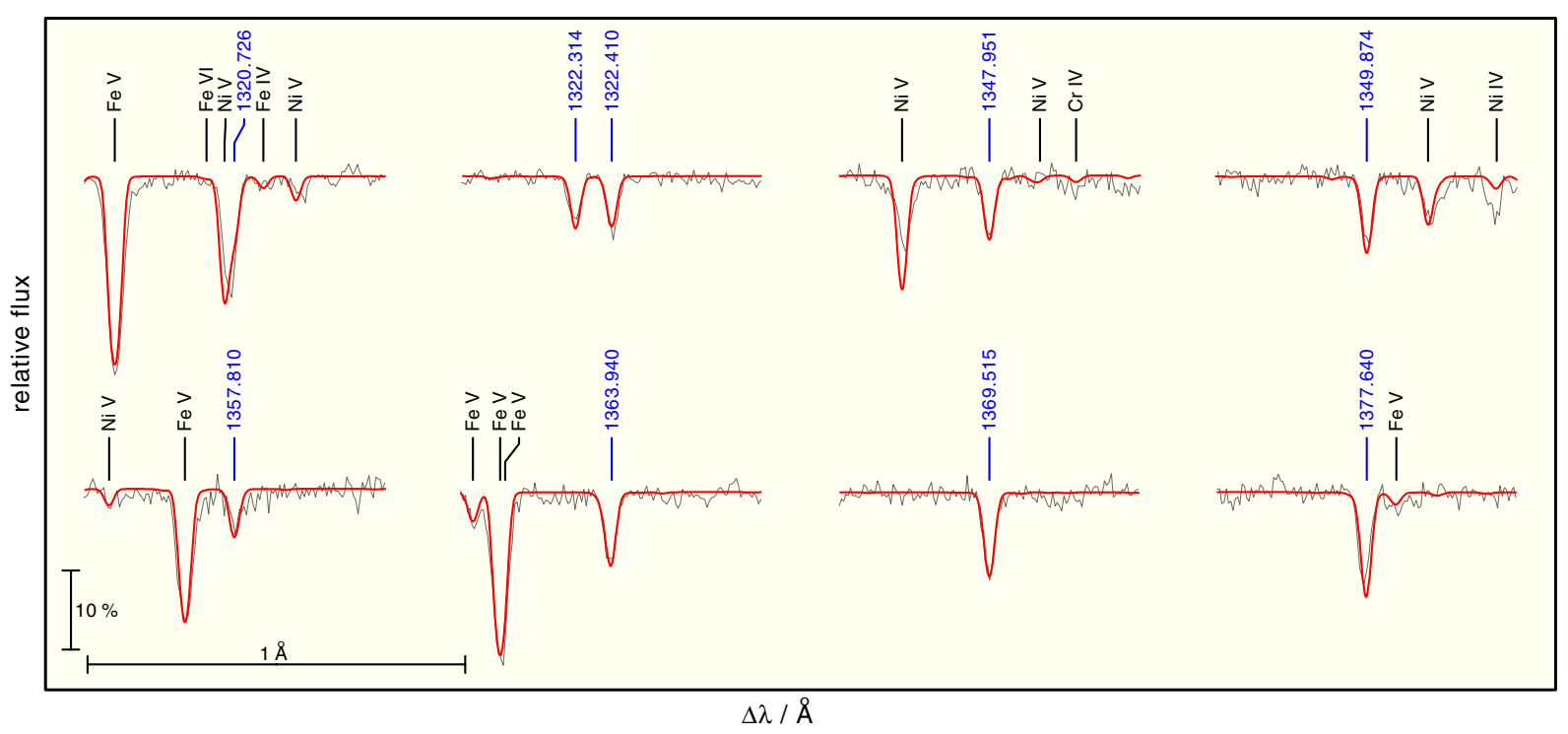

Fig. 17. Theoretical line profiles of the strongest Zn IV lines in the STIS wavelength range compared with the observation.

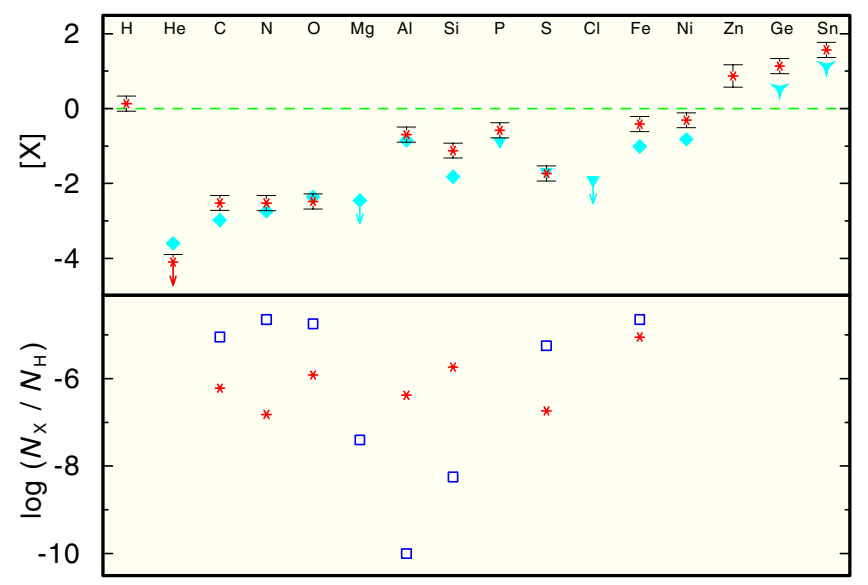

Fig. 18. Top: photospheric abundances of G191-B2B (red stars) compared with solar values (Asplund et al. 2009). [X] denotes log (mass fraction/solar mass fraction) of element $X$. The dashed, green line shows the solar ratio. The arrows indicate upper limits. The cyan diamonds (Holberg et al. 2003), triangles (Vennes et al. 1996), and tridents (Vennes et al. 2005) are previously determined values. Bottom: comparison of our abundance number ratios (red stars) with predictions of diffusion calculations for DA-type (blue squares) WDs (Chayer et al. $1995)$ with $T_{\text {eff }}=60000 \mathrm{~K}$ and $\log g=7.5$.

stratified model is, compared with the homogeneous model, no improvement. While Fe $\mathrm{V}$ lines match the observation at about $T_{\text {eff }}=55000 \mathrm{~K}$, it can be extrapolated that Fe IV lines are much too strong for $T_{\text {eff }} \lesssim 70000 \mathrm{~K}$. Ni IV and Ni V lines are much too strong because the Ni abundance is enhanced (Fig. 20) in the line-forming regions and can, thus, not be used for a $T_{\text {eff }}$ estimate.

In the stratified models, the O IV and OV lines are now mucher stronger than observed and O VI appears at the same strength that resulted from our diffusion test (Sect. 4.3). The $\mathrm{O}$ abundance profile (Fig. 20) shows a strong increase for $\log m<-4$. Only by the introduction of an artificial abundance reduction by a factor of $m / 1585$ for $\log m \lesssim-3.2$, we achieve an acceptable agreement of O V and O VI (Fig. 19). O IV is still slightly too strong because the abundance and, thus, the lines (including a blend at O IV) of the generic iron-group atom

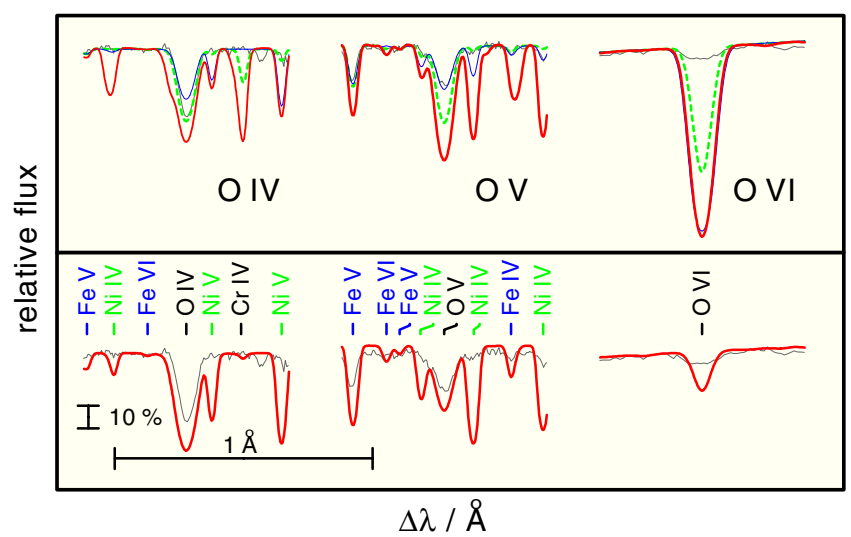

Fig. 19. Same as Fig. 14. Top: dashed, green TMAP (=chemically homogeneous) model with $O=-4.72$; thin, blue TMAP model with abundance profiles from Dreizler \& Wolff (1999), thick, red NGRT (=diffusion) model. Bottom: thick, red NGRT model with an artificially reduced $\mathrm{O}$ abundance in the outer atmosphere. Note that in the NGRT models, the line strengths of the generic iron-group element (see text) are overestimated.

(Sect. 3) are overestimated by the NGRT model (Fig. 20). Based on this numerical exercise, it may be speculated that a weak stellar wind or an other, unknown process that is not considered by NGRT is responsible for the lower oxygen abundances in the outer atmosphere.

We can conclude two things. A generic model atom is obviously not suited for a diffusion calculation due to the strongly enhanced number of lines for a single atom in the modeling process. The NGRT diffusion models yield partly too low abundances in the line-forming regions and, thus, cannot reproduce the metal line properly. An additional, weak wind may be necessary to increase the metal abundances in the line-forming regions.

\subsection{The extreme-ultraviolet spectrum}

The inability to model the EUVE spectrum with chemically homogeneous atmospheres (Holberg et al. 1989) was the reason 
T. Rauch et al.: TheoSSA: Establishing a database of synthetic stellar flux standards

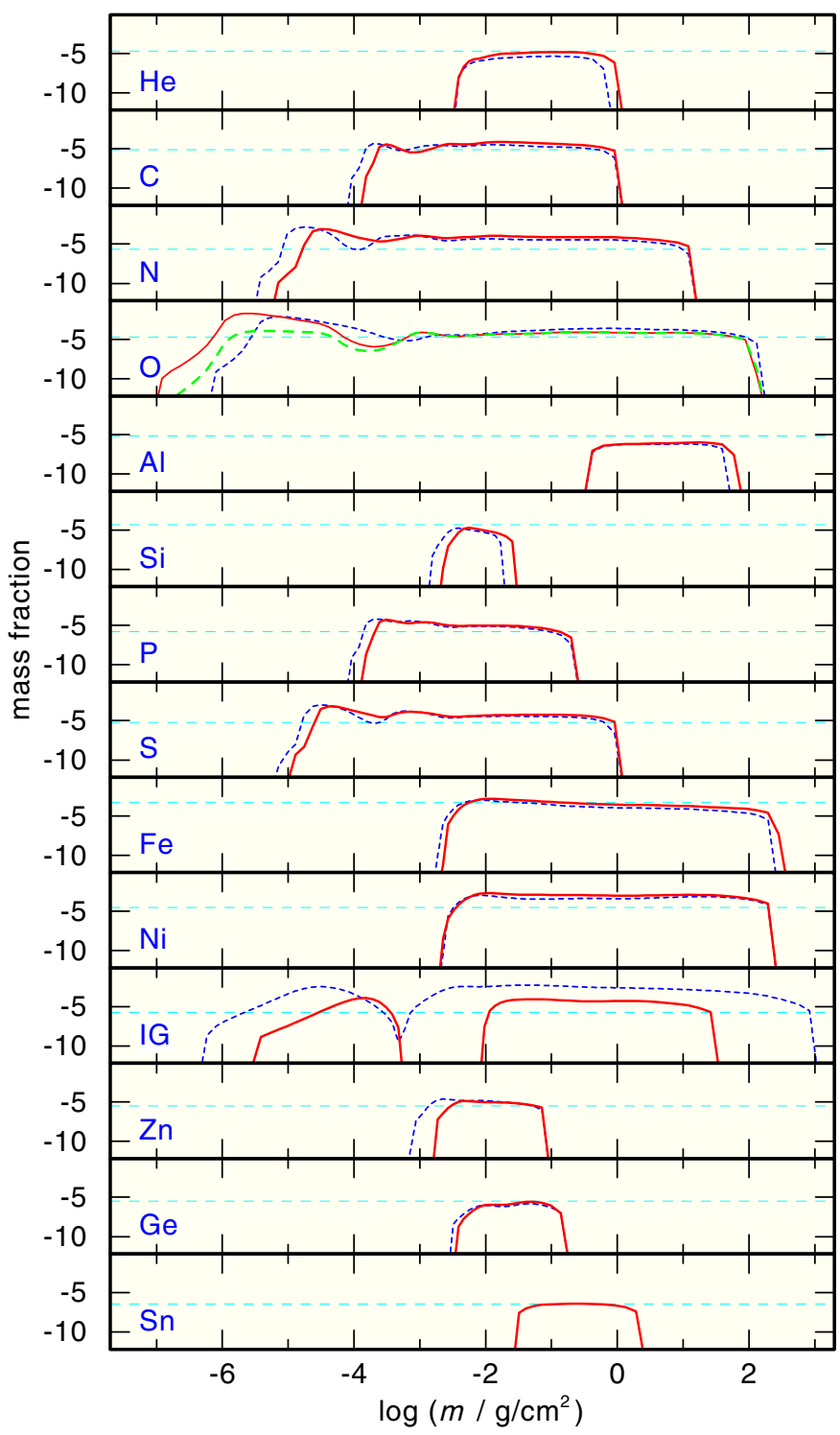

Fig. 20. Abundance profiles in our diffusion model $\left(T_{\text {eff }}=60000 \mathrm{~K}\right.$, $\log g=7.60)$. Short-dashed (blue) lines: unrealistically high abundance of the generic iron-group element (IG, see text), thick (red) lines: reduced IG abundance, horizontal long-dashed, thin (cyan) lines: the abundances in our final homogeneous model. In the $\mathrm{O}$ panel, the thick dashed (green) line shows our modified O-abundance profile (see text).

to investigate stratified photospheres (e.g. Koester 1991). Lanz et al. (1996) demonstrated, that it is possible to consistently match the optical, UV, and EUV data with homogeneous NLTE models with the same $T_{\text {eff }}$ and chemical composition.

We calculated EUV spectra from our model grid with 193584 frequency points within $100 \AA \leq \lambda \leq 930 \AA$, and Kurucz's LIN line lists (theoretical and laboratory measured lines, in total 8135405 lines of $\mathrm{Ca}-\mathrm{Ni}$ in our wavelength interval, Kurucz 2009). These spectra were processed with the recently registered VO tool TEUV ${ }^{16}$ that corrects synthetic stellar fluxes for interstellar absorption below $911 \AA$. It simulates radiative bound-free absorption of the lowest ionization states of $\mathrm{H}, \mathrm{He}, \mathrm{C}, \mathrm{N}$, and $\mathrm{O}$ using Opacity Project data (Seaton et al. 1994). Two interstellar components with different radial and turbulent velocities, temperatures, and column densities can be

\footnotetext{
${ }^{16}$ http://astro.uni-tuebingen.de/ TEUV
}

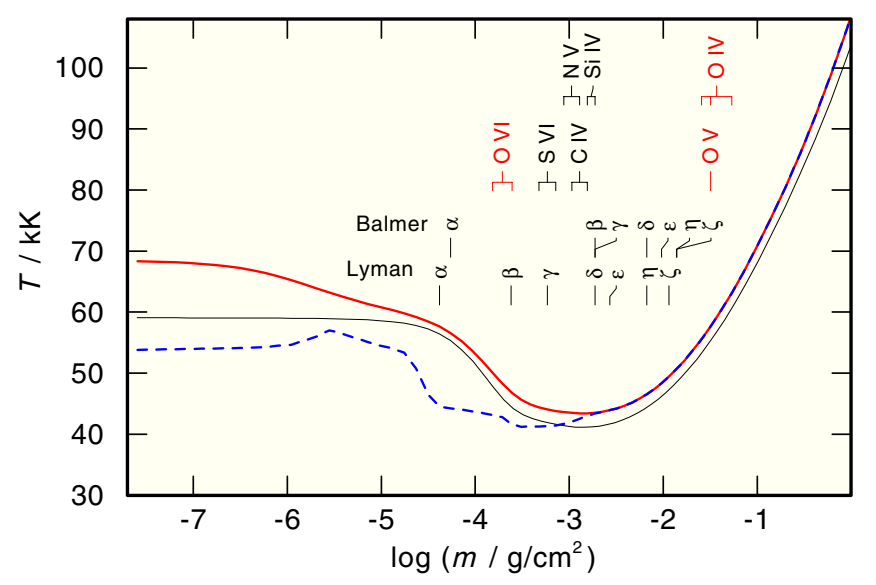

Fig. 21. Temperature structures $\left(T_{\text {eff }}=60000 \mathrm{~K}, \log g=7.60\right)$ of a pure $\mathrm{H}$ model (thin, black), our final homogeneous model (thick, red), and a diffusion model (dashed, blue). The formation depths of the line cores of the lowest members of the H I Lyman and Balmer series, the C IV, N V, O VI, Si IV, and S VI resonance doublets, and our strategic O IV $\lambda \lambda 1338.634,1343.022,1343.526 \AA$ and $\mathrm{O} v \lambda 1371.296 \AA$ lines are marked.

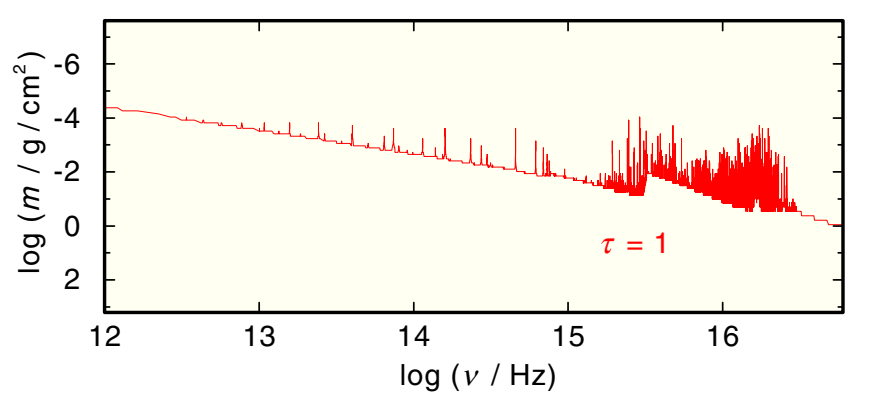

Fig. 22. Optical depth $\tau=1$ in our final homogeneous model.

considered. Figure 24 shows the comparison of synthetic and observed EUV spectra. Our synthetic spectra were normalized to the measured FUSE flux of $1.347 \times 10^{-11} \mathrm{erg} \mathrm{cm}^{-2} \mathrm{~s}^{-1} \AA^{-1}$ at $920 \AA$. Then, the interstellar column densities are adjusted, to match the EUVE flux $N_{\mathrm{H} \mathrm{I}}$ for $530 \AA, N_{\mathrm{He}}$ for $470 \AA$, and $N_{\mathrm{He} \text { II }}$ for $220 \AA$. Since our models do not reproduce the measured flux between $250 \AA$ and the He II ground state threshold, $N_{\mathrm{He}}$ is not reliable. Table 7 shows the applied $N_{\mathrm{HI}}$ and $N_{\mathrm{He}}$ values compared with the literature values. Our $N_{\mathrm{HI}}$ values, necessary to match the EUVE flux level, are about a factor of two higher than $\log \left(N_{\mathrm{HI}} / \mathrm{cm}^{2}\right)=18.34_{-0.10}^{+0.08}$ that we determined previously from H I Lyman-line fits (Sect. 2.3). $\log N_{\mathrm{N}_{\mathrm{I}}}=13.87$ and $\log N_{\mathrm{O}}=14.86$ were adopted from Lemoine et al. (2002).

The overall agreement of our homogeneous models with $T_{\text {eff }}=60000 \mathrm{~K}$ at wavelengths $\lambda \gtrsim 250 \AA$ is very good, especially the interval $360 \AA \lesssim \lambda \lesssim 450 \AA$ is excellently matched in detail. Models with $T_{\text {eff }}=65000 \mathrm{~K}$ and $T_{\text {eff }}=55000 \mathrm{~K}$ yield much too high and too low fluxes, respectively. At $\lambda \lesssim 250 \AA$ the theoretical flux is too high in all models, even at $T_{\text {eff }}=55000 \mathrm{~K}$. A stratified model (Fig. 24) with $T_{\text {eff }}=60000 \mathrm{~K}$ fails to reproduce the flux between $250 \AA \lesssim \lambda \lesssim 420 \AA$ and has a too-high flux at $\lambda \lesssim 200 \AA$.

Both, our homogeneous and our stratified models, fail to reproduce the entire EUV spectrum of G191-B2B. It seems likely that there may be some stratification in the atmosphere but we don't yet know how to distribute the various atomic species with depth. This is a challenge for theorists. 


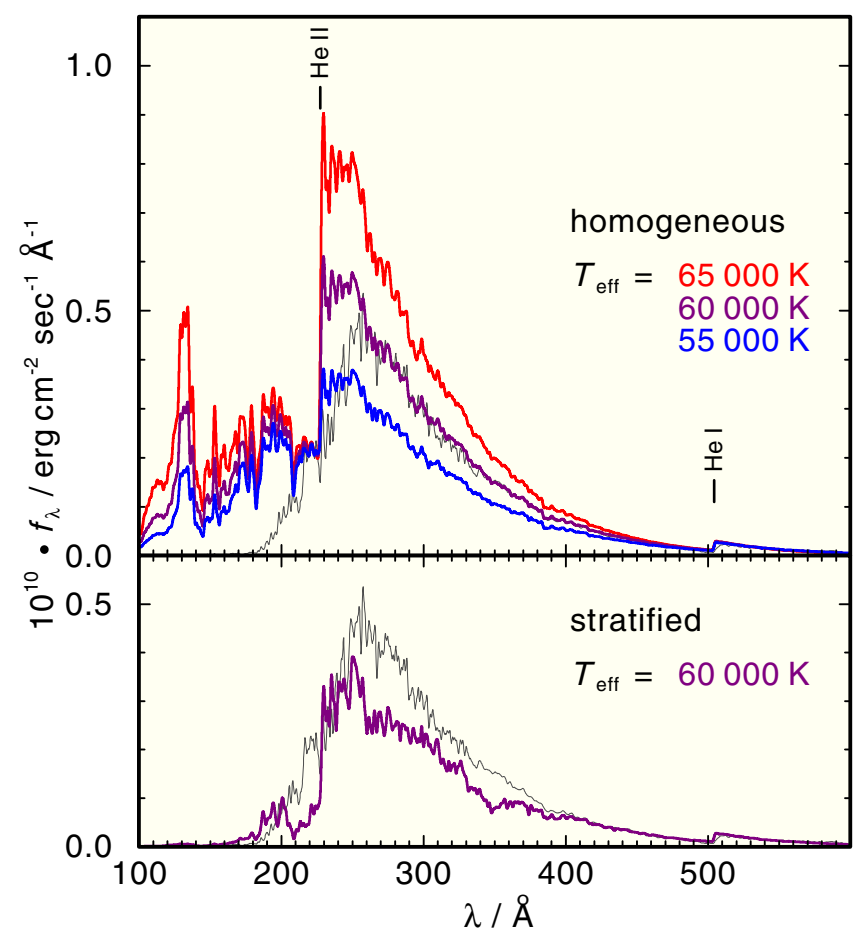

Fig. 24. Top: comparison of three synthetic spectra $\left(T_{\text {eff }}=55000\right.$, 60000 , and $65000 \mathrm{~K}$ ) of chemically homogeneous models with the EUVE observation. The wavelengths of ground-state absorption thresholds of He ions are indicated. Bottom: comparison of a stratified model $T_{\text {eff }}=60000 \mathrm{~K}$ with the observation.

Table 7. Logarithmic ISM column densities for homogeneous models with different $T_{\text {eff }}$ to match the EUVE flux level of G191-B2B.

\begin{tabular}{|c|c|c|c|}
\hline$T_{\text {eff }} / K$ & $N_{\mathrm{HI}}$ & $N_{\mathrm{HeI}}$ & \\
\hline \multicolumn{4}{|c|}{ Homogeneous (TMAP) } \\
\hline 55000 & 18.53 & 17.45 & \\
\hline 60000 & 18.59 & 17.45 & \\
\hline 65000 & 18.64 & 17.45 & \\
\hline \multicolumn{4}{|c|}{ Stratified (NGRT) } \\
\hline 60000 & 18.60 & 17.45 & \\
\hline \multicolumn{4}{|c|}{ Literature } \\
\hline 59250 & 18.23 & 17.16 & Kimble et al. (1993, HUT) \\
\hline 54000 & 18.27 & 17.16 & Dupuis et al. (1995, EUVE) \\
\hline 55200 & 18.32 & 17.26 & Lanz et al. (1996, EUVE) \\
\hline 56000 & 18.32 & 17.15 & Dreizler \& Wolff (1999, EUVE) \\
\hline 53000 & 18.28 & 17.16 & Vennes \& Lanz (2001, EUVE) \\
\hline 54000 & 18.33 & 17.34 & Cruddace et al. (2002, J-PEX) \\
\hline
\end{tabular}

\subsection{Mass and distance}

A stellar mass of $M=0.555_{-0.029}^{+0.035} M_{\odot}$ and a luminosity of $\log \left(L / L_{\odot}\right)=0.63_{-034}^{+0.37}$ are determined by comparison with evolutionary models (Fig. 25) for old white dwarfs (metallicity $z=0.001$.

We calculated the spectroscopic distance following the flux calibration of Heber et al. (1984b) for $\lambda_{\mathrm{eff}}=5454 \AA$,

$d[\mathrm{pc}]=7.11 \times 10^{-4} \cdot \sqrt{H_{v} \cdot M \times 10^{0.4 m_{\mathrm{v}_{0}}-\log g}}$,

with $m_{\mathrm{V}_{\mathrm{o}}}=m_{\mathrm{V}}-2.175 c, c=1.47 E_{B-V}$, and the Eddington flux $H_{v}=1.109 \times 10^{-3} \mathrm{erg} \mathrm{cm}^{-2} \mathrm{~s}^{-1} \mathrm{~Hz}^{-1}$ at $\lambda_{\text {eff }}$ of our final model atmosphere. We used $E_{B-V}=0.0005 \pm 0.0005$ (Sect. 2.3), $M=0.555_{-0.029}^{+0.035} M_{\odot}$, and $m_{\mathrm{V}}=11.7228 \pm 0.0082$ (van Leeuwen 2007), and derived a distance of $d=62 \pm 4 \mathrm{pc}$ and a height above

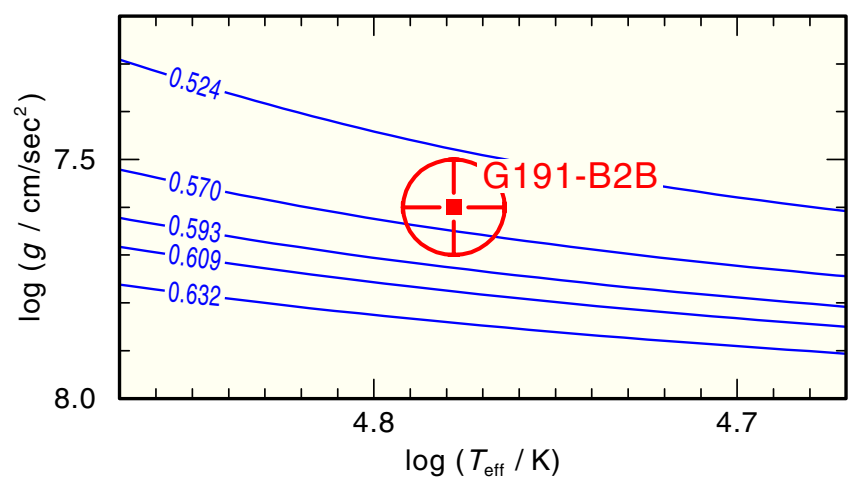

Fig. 25. Location of G191-B2B in the $\log T_{\text {eff }}-\log g$ plane (the ellipse indicates the errors of our analysis) compared with evolutionary tracks for hydrogen-rich white dwarfs (Renedo et al. 2010) labeled with the respective stellar masses (in $M_{\odot}$ ).

Table 8. Parameters of G191-B2B as derived by our analysis.

\begin{tabular}{|c|c|c|c|}
\hline $\begin{array}{l}T_{\text {eff }} / \mathrm{K} \\
\log \left(g / \mathrm{cm} / \mathrm{s}^{2}\right)\end{array}$ & $\begin{array}{r}60000 \pm 2000 \\
7.60 \pm 0.05 \\
\end{array}$ & & \\
\hline \multirow{2}{*}{ Element } & Mass & Number & \multirow{2}{*}[X]{} \\
\hline & \multicolumn{2}{|l|}{ fraction } & \\
\hline $\mathrm{H}$ & $9.99 \times 10^{-1}$ & 1.0 & 0.132 \\
\hline $\mathrm{He}$ & $<1.98 \times 10^{-5}$ & $<5.0 \times 10^{-6}$ & $<-4.099$ \\
\hline $\mathrm{C}$ & $7.15 \times 10^{-6}$ & $6.0 \times 10^{-7}$ & -2.520 \\
\hline $\mathrm{N}$ & $2.08 \times 10^{-6}$ & $1.5 \times 10^{-7}$ & -2.522 \\
\hline $\mathrm{O}$ & $1.90 \times 10^{-5}$ & $1.2 \times 10^{-6}$ & -2.479 \\
\hline $\mathrm{Al}$ & $1.12 \times 10^{-5}$ & $4.2 \times 10^{-7}$ & -0.695 \\
\hline $\mathrm{Si}$ & $5.01 \times 10^{-5}$ & $1.8 \times 10^{-6}$ & -1.123 \\
\hline$P$ & $1.54 \times 10^{-6}$ & $5.0 \times 10^{-8}$ & -0.579 \\
\hline S & $5.72 \times 10^{-6}$ & $1.8 \times 10^{-7}$ & -1.733 \\
\hline $\mathrm{Ti}$ & $<3.13 \times 10^{-7}$ & $<1.1 \times 10^{-7}$ & $<-0.100$ \\
\hline $\mathrm{Cr}$ & $<1.66 \times 10^{-6}$ & $<5.1 \times 10^{-7}$ & $<-0.100$ \\
\hline $\mathrm{Mn}$ & $<1.08 \times 10^{-6}$ & $<3.2 \times 10^{-7}$ & $<-0.100$ \\
\hline $\mathrm{Fe}$ & $4.98 \times 10^{-4}$ & $9.0 \times 10^{-6}$ & -0.414 \\
\hline Co & $<4.92 \times 10^{-7}$ & $<1.2 \times 10^{-7}$ & $<-0.100$ \\
\hline $\mathrm{Ni}$ & $3.49 \times 10^{-5}$ & $6.0 \times 10^{-7}$ & -0.310 \\
\hline $\mathrm{Zn}$ & $1.30 \times 10^{-5}$ & $2.0 \times 10^{-7}$ & 0.873 \\
\hline $\mathrm{Ge}$ & $3.24 \times 10^{-6}$ & $4.5 \times 10^{-8}$ & 1.135 \\
\hline Sn & $3.53 \times 10^{-7}$ & $3.0 \times 10^{-9}$ & 1.569 \\
\hline$E_{B-V}$ & $0.0005 \pm 0.0005$ & & \\
\hline $\log \left(N_{\mathrm{HI}} / \mathrm{cm}^{2}\right)$ & $18.34_{-0.10}^{+0.08}$ & & \\
\hline $\log \left(N_{\text {DI }} / \mathrm{cm}^{2}\right)$ & $13.54_{-0.06}^{+0.05}$ & & \\
\hline$d / \mathrm{pc}$ & $62 \pm 4^{-0 .}$ & & \\
\hline$M / M_{\odot}$ & $0.555_{-0.029}^{+0.035}$ & & \\
\hline$R / R_{\odot}$ & $0.0195_{-0.0005}^{+0.0004}$ & & \\
\hline $\log \left(L / L_{\odot}\right)$ & $0.63_{-0.34}^{+0.37}$ & & \\
\hline
\end{tabular}

the Galactic plane of $z=8 \pm 1$ pc. This is in agreement with the HIPPARCos ${ }^{17}$ parallax measurement (van Leeuwen 2007, HIP23692) of $d=59.88_{-12.95}^{+9.05} \mathrm{pc}$ and the XHIP ${ }^{18}$ value of $d=57.96 \pm 10.31 \mathrm{pc}$ (Anderson \& Francis 2012). The spectroscopic distance of $d=55.84 \pm 0.86 \mathrm{pc}$ determined by Holberg et al. (2008) is slightly smaller, this error estimate, however, appears to be too optimistic.

\footnotetext{
17 http://www.rssd.esa.int/index.php?project=HIPPARCOS ${ }^{18}$ Extended HIPPARcos compilation
} 
T. Rauch et al.: TheoSSA: Establishing a database of synthetic stellar flux standards

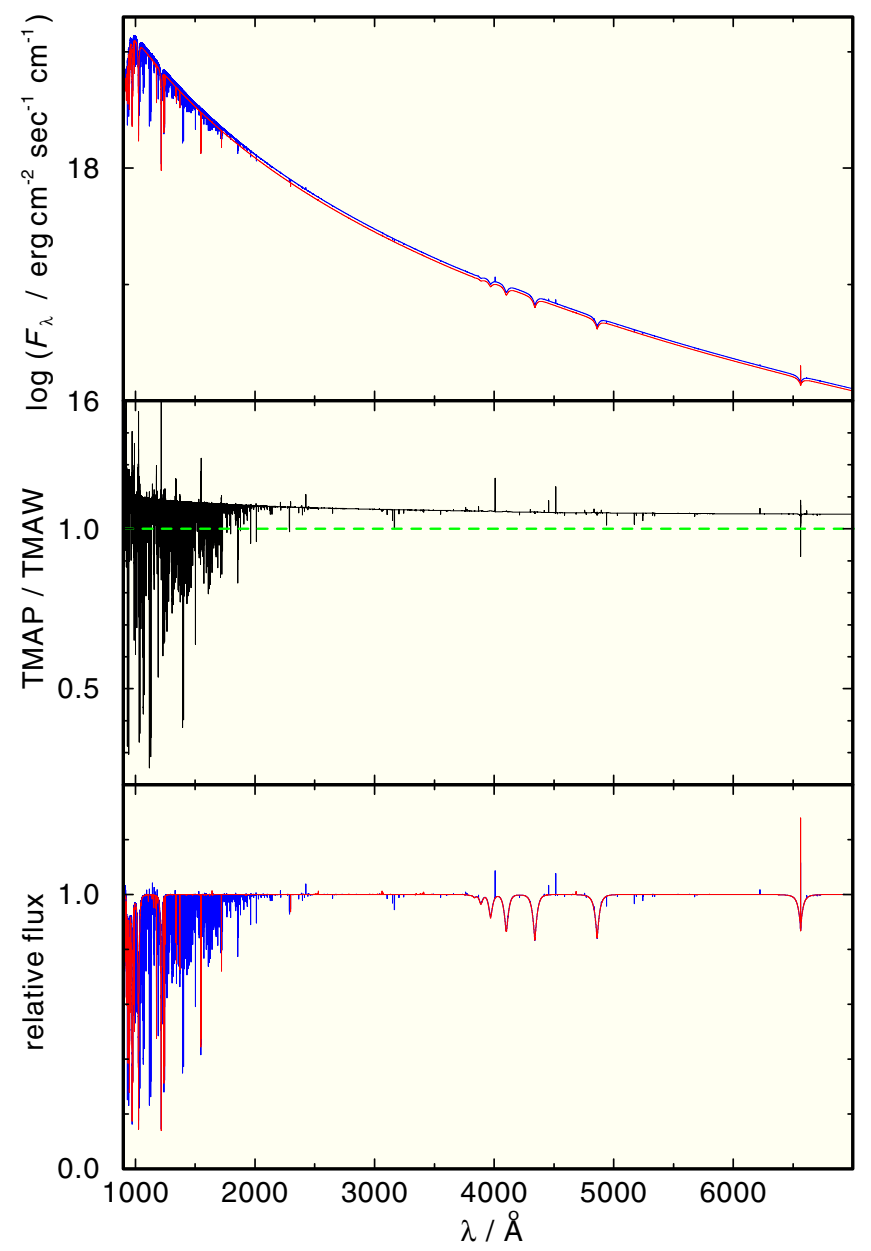

Fig. 26. Comparison of our final TMAP model (blue, thick) with a TMAW model (red, thin). Top: astrophysical fluxes at the stellar surface, middle: ratio TMAP/TMAW flux, bottom: normalized fluxes.

\section{TheoSSA: synthetic stellar spectra on demand}

At the end, we want to compare our final TMAP model flux with an SED, that was calculated with the TMAW tool (Sect. 1) and considers $\mathrm{H}, \mathrm{He}, \mathrm{C}, \mathrm{N}$, and $\mathrm{O}$ only. Figure 26 shows that the TMAP flux is higher everywhere at $\lambda>911 \AA$. The reason is strong metal-line blanketing at $\lambda<911 \AA$ that causes a flux increase at longer wavelengths. It amounts to about $10 \%$ at $1000 \AA$ and to about $5 \%$ at $7000 \AA$. The lower panel of Fig. 26 illustrates that the theoretical line profiles of the H I Balmer series are almost identical in both, TMAP and TMAW models, with the exception of an increased emission reversal in the line core of $\mathrm{H} \alpha$ in the TMAP model.

The TheoSSA database contains currently TMAP SEDs of a dozen standard stars. Some of them are represented by different models for comparison, because they were initially calculated for the calibration (Vernet et al. 2008a,b, 2010) of ESO's ${ }^{19}$ second generation VLT $^{20}$ instrument XSHOOTER ${ }^{21}$ (Vernet et al. 2011) while the parameters of Gianninas et al. (2011) and Giammichele et al. (2012) were published later (Table 9).

\footnotetext{
${ }^{19}$ European Southern Observatory.

${ }^{20}$ Very Large Telescope.

${ }^{21}$ http://www . eso.org/sci/facilities/paranal/ instruments/xshooter
}

Table 9. Standard star SEDs (references for $T_{\text {eff }}$ and $\log g$ are given) presently available in TheoSSA.

\begin{tabular}{|c|c|c|c|c|}
\hline Name & WD no. & Spectral type & $\begin{array}{l}T_{\text {eff }} \\
{[\mathrm{K}]} \\
\end{array}$ & $\begin{array}{c}\log g \\
{\left[\mathrm{~cm} / \mathrm{s}^{2}\right]}\end{array}$ \\
\hline EG 274 & $1620-391$ & $\mathrm{DA} 2(+\mathrm{G} 5 \mathrm{~V})$ & $\begin{array}{l}24276 \\
25980\end{array}$ & $\begin{array}{l}8.01^{a} \\
7.96^{b, c}\end{array}$ \\
\hline Feige 67 & & Op+WDsd & 75000 & $5.20^{d}$ \\
\hline Feige 110 & $2317-054$ & $\mathrm{sdO}$ & 40000 & $5.00^{e}$ \\
\hline G191-B2B & $0501+527$ & DA0 & $\begin{array}{l}58883 \\
61193 \\
60920 \\
60000\end{array}$ & $\begin{array}{l}7.46^{a} \\
7.49^{f} \\
7.55^{b} \\
7.55^{g}\end{array}$ \\
\hline G 93-48 & $2149+021$ & DAZ3 & $\begin{array}{l}18100 \\
18170\end{array}$ & $\begin{array}{l}7.85^{h} \\
8.01^{b}\end{array}$ \\
\hline GD 50 & 0346-011 & DA2 & $\begin{array}{l}40550 \\
42700\end{array}$ & $\begin{array}{l}9.22^{i} \\
9.20^{b}\end{array}$ \\
\hline GD 71 & $0549+158$ & DA1 & $\begin{array}{l}32747 \\
32780 \\
33590\end{array}$ & $\begin{array}{l}7.68^{f} \\
7.83^{i} \\
7.93^{b}\end{array}$ \\
\hline GD 108 & $0958-073$ & $\mathrm{sdB}$ & 22908 & $5.30^{j}$ \\
\hline GD 153 & $1254+223$ & DA1.5 & $\begin{array}{l}38205 \\
38686 \\
40590\end{array}$ & $\begin{array}{l}7.89^{i} \\
7.66^{f} \\
7.93^{b}\end{array}$ \\
\hline $\mathrm{HZ} 2$ & $0410+117$ & DA3 & $\begin{array}{l}20600 \\
21600\end{array}$ & $\begin{array}{l}7.90^{h} \\
7.98^{b}\end{array}$ \\
\hline $\mathrm{HZ} 43 \mathrm{~A}$ & $1314+293$ & $\mathrm{DA} 1+\mathrm{dM} 3 \mathrm{e}$ & $\begin{array}{l}51116 \\
56800\end{array}$ & $\begin{array}{l}7.90^{k} \\
7.89^{b}\end{array}$ \\
\hline Sirius B & $0642-166$ & DA2 & $\begin{array}{l}24826 \\
25970\end{array}$ & $\begin{array}{l}8.60^{k} \\
8.57^{b, c}\end{array}$ \\
\hline
\end{tabular}

Notes. WD numbers are from McCook \& Sion (1999). ${ }^{(a)}$ Assumed; (b) Gianninas et al. (2011); ${ }^{(c)}$ Giammichele et al. (2012); ${ }^{(d)}$ Bauer \& Husfeld (1995); ${ }^{(e)}$ Heber et al. (1984a), He mass fraction of 0.107; ${ }^{(f)}$ Finley et al. (1997); ${ }^{(g)}$ this work; ${ }^{(h)}$ Guseinov et al. (1983); (i) Barstow et al. (2001); ${ }^{(j)}$ Kilkenny et al. (1988); ${ }^{(k)}$ Beuermann et al. (2006).

\section{Accuracy of flux calibration with G191-B2B}

Our spectral analysis was performed using state-of-the-art atomic data and model-atmosphere code. The best reproduction of UV and optical spectra was achieved with chemically homogeneous models. In Fig. 27, we compared two models at the edge of our error ranges in $T_{\text {eff }}$ and $\log g$. The deviation in the continuum flux of two TMAP model SEDs is $\approx 3 \%$ in the optical and $\approx 5 \%$ in the FUV. A systematic error is present due to the uncertainty of the used atomic data, such as oscillator strengths where it is typically $\approx 15 \%$ for a single line. The employment of many lines of many ions of many atoms in a spectral analysis minimizes the propagation of these uncertainties into the errors of the main photospheric properties like $T_{\text {eff }}, \log g$, and the abundances. An additional systematic error may be present between individual model-atmosphere packages (e.g. Rauch 2008b) because of differences in coding, approximations, etc.

The situation for the flux calibration is, however, not that strongly dependent on the exact $T_{\text {eff }}$ and $\log g$ values (the latter is even less important). E.g. in the case of G191-B2B and our errors (3\% in $T_{\text {eff }}$ and $0.05 \mathrm{dex}$ in $\log g$ ), a normalization to a precisely measured brightness will reduce the deviation between model SED and observation much below $1 \%$ in the optical and infrared. The residuals among the three primary stars G191-B2B, GD 71, and GD 153 are generally sub percent at the longer wavelengths (Bohlin 2007). The remaining deviation in 


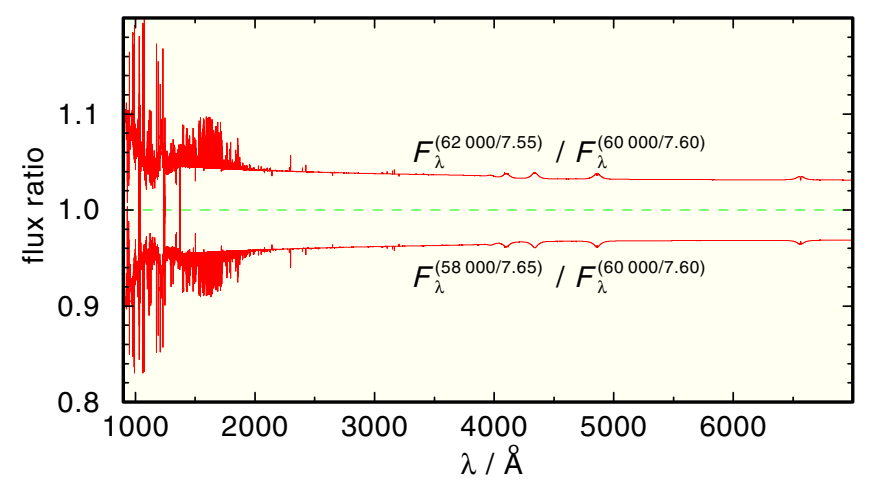

Fig. 27. Flux ratio of two TMAP models at the edge of the $T_{\text {eff }}$ and $\log g$ error ranges and our final model.
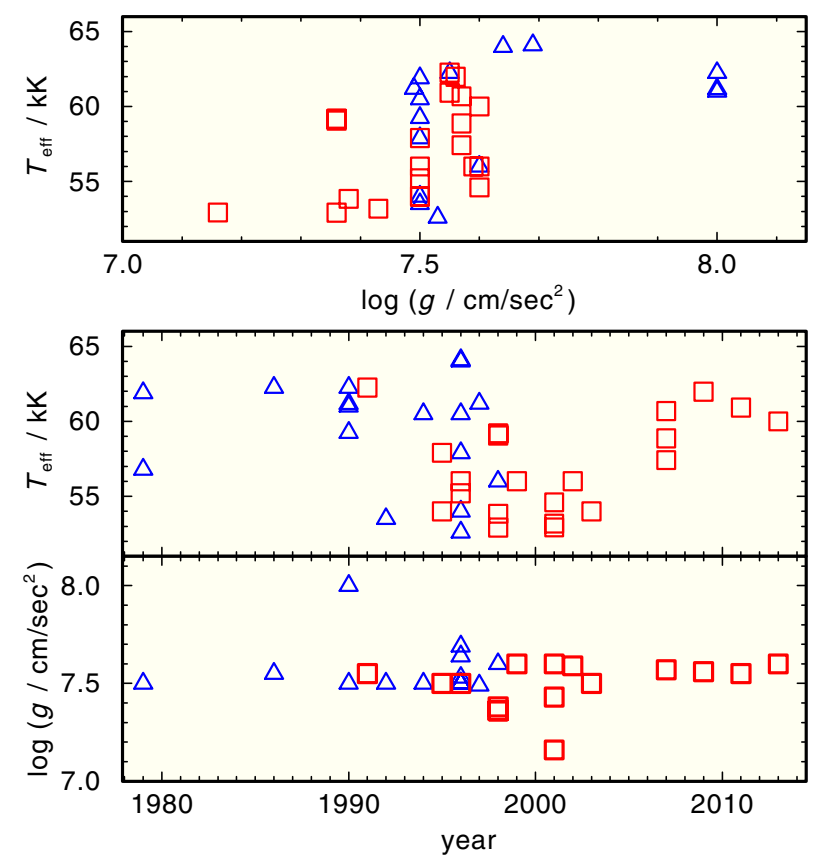

Fig. 28. Determined $T_{\text {eff }}$ and $\log g$ values in the last 34 years. Blue triangles denote analyses with LTE models, red squares those with NLTE models (Table 2). The result of Koester et al. $(1979, \log g=5.95)$ is outside the top and bottom panels.

the FUV wavelength range is presently less than 2\%. Further improvement is essentially dependent on the reliability of the atomic data (Sect. 7).

\section{Conclusions}

The TheoSSA service is designed to provide theoretical stellar SEDs of any kind in VO-compliant form. Its efficiency is strongly increasing if more different model-atmosphere groups provide their SEDs with a proper description in their respective meta data. The establishment of a database of spectrophotometric standard stars is an opportunity to use the same model SEDs for astrophysical flux calibration. Many modelatmosphere groups have their own best models for some of these stars, for which a common base for comparison arises. Differences in the algorithms for considered physics, assumptions, and approximations in different model-atmosphere codes, lead to systematic deviations in general.

Figure 28 shows the temporal development of the $T_{\text {eff }}$ and $\log g$ determinations of G191-B2B. While both values had a large scatter in the 1990s (error ranges are not shown for clarity), the three most recent analyses, that are all based on sophisticated NLTE model-atmosphere techniques, show a good agreement within relatively narrow error ranges of about $3 \%$ in $T_{\text {eff }}$ and $0.05 \operatorname{dex}(\approx 12 \%)$ in $\log g$. Ironically, these latest results agree quite well with the very first line-profile analysis presented by Holberg et al. (1986) performed with a Ly $\alpha$ line-profile fit with a simple, pure-H LTE model atmosphere. The TheoSSA database may help to get closer to the intended goal of $1 \%$ accuracy in absolute flux calibration.

We presented here our spectral analysis of G191-B2B to demonstrate the current state-of-the-art. We are presently able to reproduce the observed spectrum from $250 \AA$ to the infrared. The EUV part from $150 \AA$ to $250 \AA$ cannot be modeled, neither by our homogeneous nor by our stratified models. The reason is unknown.

A similar analysis of the UV spectrum of the calibration star $\mathrm{BD}+28^{\circ} 4211\left(T_{\text {eff }}=82000 \pm 5000 \mathrm{~K}, \log g=6.2_{-0.1}^{+0.3}\right)$ was just published by Latour et al. (2013).

Model-atmosphere codes have arrived now at a high level of sophistication, and we already encounter problems getting reliable atomic data to reproduce the high-resolution and high$\mathrm{S} / \mathrm{N}$ spectra that are obtained with presently available instruments. This is a challenge for atomic physicists to be prepared for upcoming telescopes and instruments.

Acknowledgements. TR is supported by the German Aerospace Center (DLR, grant 05 OR 1301). The GAVO project at Tübingen has been supported by the Federal Ministry of Education and Research (BMBF, grants 05 AC 6 VTB, 05 AC 11 VTB). AstroGrid-D was funded by the BMBF (01 AK 804 [A-G]). The bwGRiD ${ }^{22}$ is funded within the framework of the D-Grid Project by the BMBF. This research has made use of the SIMBAD database, operated at the CDS, Strasbourg, France. This research has made use of NASA's Astrophysics Data System. This work used the WRPLOT visualization software developed by Wolf-Rainer Hamann (Potsdam) and the WRPLOT team. Some of the data presented in this paper were obtained from the Mikulski Archive for Space Telescopes (MAST). STScI is operated by the Association of Universities for Research in Astronomy, Inc., under NASA contract NAS5-26555. Support for MAST for non-HST data is provided by the NASA Office of Space Science via grant NNX09AF08G and by other grants and contracts. The TEUV tool (http://astro-uni-tuebingen.de/ TEUV) used to apply interstellar corrections to theoretical spectra was constructed as part of the activities of the German Astrophysical Virtual Observatory. The TIRO service (http:// astro-uni-tuebingen.de/ TIRO) used to calculate opacities for this paper was constructed as part of the activities of the German Astrophysical Virtual Observatory.

\section{References}

Allende Prieto, C., Hubeny, I., \& Smith, J. A. 2009, MNRAS, 396, 759 Anderson, E., \& Francis, C. 2012, Astron. Lett., 38, 331

Asplund, M., Grevesse, N., Sauval, A. J., \& Scott, P. 2009, ARA\&A, 47, 481

Barstow, M. A., \& Hubeny, I. 1998, MNRAS, 299, 379

Barstow, M. A., Hubeny, I., \& Holberg, J. B. 1998, MNRAS, 299, 520

Barstow, M. A., Holberg, J. B., Hubeny, I., et al. 2001, MNRAS, 328, 211

Barstow, M. A., Good, S. A., Holberg, J. B., et al. 2003, MNRAS, 341, 870

Barstow, M. A., Cruddace, R. G., Kowalski, M. P., et al. 2005, MNRAS, 362 1273

Bauer, F., \& Husfeld, D. 1995, A\&A, 300, 481

Berengut, J. C., Flambaum, V. V., Ong, A., et al. 2013, Phys. Rev. Lett., 111, 010801

Beuermann, K., Burwitz, V., \& Rauch, T. 2006, A\&A, 458, 541

Beuermann, K., Burwitz, V., \& Rauch, T. 2008, A\&A, 481, 769

Bohlin, R. C. 2007, in The Future of photometric, spectrophotometric and polarimetric standardization, ed. C. Sterken, ASP Conf. Ser., 364, 315

Bohlin, R. C., Dickinson, M. E., \& Calzetti, D. 2001, AJ, 122, 2118

Chayer, P., Fontaine, G., \& Wesemael, F. 1995, ApJS, 99, 189

Chayer, P., Vennes, S., Pradhan, A. K., et al. 1996, in Astrophysics in the extreme ultraviolet, eds. S. Bowyer, \& R. F. Malina, IAU Colloq. 152, 211

22 http://www.bw-grid.de/en/the-bwgrid/ 
T. Rauch et al.: TheoSSA: Establishing a database of synthetic stellar flux standards

Cruddace, R. G., Kowalski, M. P., Yentis, D., et al. 2002, ApJ, 565, 47

Cutri, R. M., Skrutskie, M. F., van Dyk, S., et al. 2003, VizieR Online Data Catalog, II/246

Dickinson, N. J., Barstow, M. A., \& Hubeny, I. 2012a, MNRAS, 421, 3222

Dickinson, N. J., Barstow, M. A., Welsh, B. Y., et al. 2012b, MNRAS, 423, 1397

Dreizler, S., \& Wolff, B. 1999, A\&A, 348, 189

Dupuis, J., Vennes, S., Bowyer, S., Pradhan, A. K., \& Thejll, P. 1995, ApJ, 455, 574

Finley, D. S., Basri, G., \& Bowyer, S. 1990, ApJ, 359, 483

Finley, D. S., Koester, D., \& Basri, G. 1997, ApJ, 488, 375

Fitzpatrick, E. L. 1999, PASP, 111, 63

Giammichele, N., Bergeron, P., \& Dufour, P. 2012, ApJS, 199, 29

Gianninas, A., Bergeron, P., \& Ruiz, M. T. 2011, ApJ, 743, 138

Green, J., Jelinsky, P., \& Bowyer, S. 1990, ApJ, 359, 499

Groenewegen, M. A. T., \& Lamers, H. J. G. L. M. 1989, A\&AS, 79, 359

Gunderson, K., Wilkinson, E., Green, J. C., \& Barstow, M. A. 2001, ApJ, 562, 992

Guseinov, O. K., Novruzova, K. I., \& Rustamov, I. S. 1983, Ap\&SS, 96, 1

Heber, U., Hamann, W.-R., Hunger, K., et al. 1984a, A\&A, 136, 331

Heber, U., Hunger, K., Jonas, G., \& Kudritzki, R. P. 1984b, A\&A, 130, 119

Heber, U., Moehler, S., Napiwotzki, R., Thejll, P., \& Green, E. M. 2002, A\&A, 383,938

Høg, E., Fabricius, C., Makarov, V. V., et al. 2000, A\&A, 355, L27

Holberg, J. B., Wesemael, F., \& Basile, J. 1986, ApJ, 306, 629

Holberg, J. B., Kidder, K., Liebert, J., \& Wesemael, F. 1989, in White dwarfs,

Lect. Notes Phys. 328, ed. G. Wegner (Berlin: Springer Verlag), IAU Colloq., 114,188

Holberg, J. B., Ali, B., Carone, T. E., \& Polidan, R. S. 1991, ApJ, 375, 716

Holberg, J. B., Hubeny, I., Barstow, M. A., et al. 1994, ApJ, 425, L105

Holberg, J. B., Barstow, M. A., \& Sion, E. M. 1998, ApJS, 119, 207

Holberg, J. B., Barstow, M. A., Hubeny, I., et al. 2003, in Hubble's science legacy: future optical/ultraviolet astronomy from space, eds. K. R. Sembach, J. C. Blades, G. D. Illingworth, \& R. C. Kennicutt, Jr., ASP Conf. Ser., 291, 383

Holberg, J. B., Sion, E. M., Oswalt, T., et al. 2008, AJ, 135, 1225

Hubeny, I., \& Lanz, T. 1995, ApJ, 439, 875

Hubeny, I., Hummer, D. G., \& Lanz, T. 1994, A\&A, 282, 151

Hummer, D. G., \& Mihalas, D. 1988, ApJ, 331, 794

Kilkenny, D., Heber, U., \& Drilling, J. S. 1988, South African Astronomical Observatory Circular, 12, 1

Kimble, R. A., Davidsen, A. F., Blair, W. P., et al. 1993, ApJ, 404, 663

Koester, D. 1991, in Evolution of stars: the photospheric abundance connection, eds. G. Michaud, \& A. V. Tutukov, IAU Symp., 145, 435

Koester, D., \& Finley, D. 1992, in The Atmospheres of early-type stars, eds. U. Heber, \& C. S. Jeffery (Berlin: Springer Verlag), Lect. Notes Phys., 401, 314

Koester, D., Schulz, H., \& Weidemann, V. 1979, A\&A, 76, 262

Kurucz, R. L. 1991, in NATO ASIC Proc. 341: Stellar atmospheres - beyond classical models, eds. L. Crivellari, I. Hubeny, \& D. G. Hummer (Dordrecht: Reidel Publishing Co.), 441

Kurucz, R. L. 2009, in Am. Inst. Phys. Conf. Ser., eds. I. Hubeny, J. M. Stone, K. MacGregor, \& K. Werner, 1171, 43

Kurucz, R. L. 2011, Can. J. Phys., 89, 417

Kwok, S., Purton, C. R., \& Fitzgerald, P. M. 1978, ApJ, 219, L125

Lajoie, C.-P., \& Bergeron, P. 2007, ApJ, 667, 1126

Landolt, A. U., \& Uomoto, A. K. 2007, AJ, 133, 768

Lanz, T., Barstow, M. A., Hubeny, I., \& Holberg, J. B. 1996, ApJ, 473, 1089

Latour, M., Fontaine, G., Chayer, P., \& Brassard, P. 2013, ApJ, 773, 84

Lemoine, M., Vidal-Madjar, A., Hébrard, G., et al. 2002, ApJS, 140, 67

Liebert, J., Bergeron, P., \& Holberg, J. B. 2005, ApJS, 156, 47

McCook, G. P., \& Sion, E. M. 1999, ApJS, 121, 1
Morton, D. C. 2000 , ApJS, 130, 403

Napiwotzki, R., \& Rauch, T. 1994, A\&A, 285, 603

Oegerle, W. R., Jenkins, E. B., Shelton, R. L., Bowen, D. V., \& Chayer, P. 2005, ApJ, 622, 377

Pancino, E., Altavilla, G., Marinoni, S., et al. 2012, MNRAS, 426, 1767

Preval, S. P., Barstow, M. A., Holberg, J. B., \& Dickinson, N. J. 2013, in ASP Conf. Ser. 469, eds. J. Krzesiński, G. Stachowski, P. Moskalik, \& K. Bajan, 193

Rauch, T. 1997, A\&A, 320, 237

Rauch, T. 2003, A\&A, 403, 709

Rauch, T. 2008a, in Proc. Astronomical Spectroscopy and Virtual Observatory, eds. M. Guainazzi, \& P. Osuna (European Space Agency), 183

Rauch, T. 2008b, A\&A, 481, 807

Rauch, T. 2012 [arXiv: 1210.7636]

Rauch, T., \& Deetjen, J. L. 2003, in Stellar atmosphere modeling, eds. I. Hubeny, D. Mihalas, \& K. Werner, ASP Conf. Ser., 288, 103

Rauch, T., \& Nickelt, I. 2009, in Multi-wavelength Astronomy and Virtual Observatory, eds. D. Baines, \& P. Osuna, 49

Rauch, T., \& Ringat, E. 2011, in Astronomical data analysis software and systems XX, eds. I. N. Evans, A. Accomazzi, D. J. Mink, \& A. H. Rots, ASP Conf. Ser., 442, 563

Rauch, T., Ziegler, M., Werner, K., et al. 2007, A\&A, 470, 317

Rauch, T., Nickelt, I., Stampa, U., Demleitner, M., \& Koesterke, L. 2009, in Astronomical data analysis software and systems XVIII, eds. D. A. Bohlender, D. Durand, \& P. Dowler, ASP Conf. Ser., 411, 388

Rauch, T., Ringat, E., \& Werner, K. 2010 [arXiv: 1011.3628]

Rauch, T., Werner, K., Biémont, É., Quinet, P., \& Kruk, J. W. 2012, A\&A, 546, A55

Renedo, I., Althaus, L. G., Miller Bertolami, M. M., et al. 2010, ApJ, 717, 183

Ringat, E., \& Rauch, T. 2010, in Am. Inst. Phys. Conf. Ser., 1273, eds. K. Werner, \& T. Rauch, 121

Ringat, E., Rauch, T., \& Werner, K. 2012, Baltic Astron., 21, 341

Sahu, M. S., Landsman, W., Bruhweiler, F. C., et al. 1999, ApJ, 523, L159

Savitzky, A., \& Golay, M. J. E. 1964, Anal. Chem., 36, 1627

Schuh, S. L., Dreizler, S., \& Wolff, B. 2002, A\&A, 382, 164

Seaton, M. J., Yan, Y., Mihalas, D., \& Pradhan, A. K. 1994, MNRAS, 266, 805

Shipman, H. L. 1979, ApJ, 228, 240

Tremblay, P.-E., \& Bergeron, P. 2009, ApJ, 696, 1755

van Leeuwen, F. 2007, A\&A, 474, 653

Vennes, S., \& Lanz, T. 2001, ApJ, 553, 399

Vennes, S., Thejll, P., \& Shipman, H. L. 1991, in NATO ASIC Proc. 336, White dwarfs, eds. G. Vauclair, \& E. Sion, 235

Vennes, S., Chayer, P., Hurwitz, M., \& Bowyer, S. 1996, ApJ, 468, 898

Vennes, S., Polomski, E. F., Lanz, T., et al. 2000, ApJ, 544, 423

Vennes, S., Chayer, P., \& Dupuis, J. 2005, ApJ, 622, L121

Vernet, J., Kerber, F., D’Odorico, S., et al. 2008a, in 2007 ESO Instrument Calibration Workshop, eds. A. Kaufer, \& F. Kerber (Heidelberg, Berlin: Spring Verlag), 153

Vernet, J., Kerber, F., Saitta, F., et al. 2008b, in Proc. SPIE, 7016

Vernet, J., Kerber, F., Mainieri, V., et al. 2010, Highlights of Astronomy, 15, 535

Vernet, J., Dekker, H., D’Odorico, S., et al. 2011, A\&A, 536, A105

Vidal-Madjar, A., Allard, N. F., Koester, D., et al. 1994, A\&A, 287, 175

Vidal-Madjar, A., Lemoine, M., Ferlet, R., et al. 1998, A\&A, 338, 694

Wassermann, D., Werner, K., Rauch, T., \& Kruk, J. W. 2010, A\&A, 524, A9

Werner, K., \& Dreizler, S. 1994, A\&A, 286, L31

Werner, K., Deetjen, J. L., Dreizler, S., et al. 2003, in Stellar atmosphere modeling, eds. I. Hubeny, D. Mihalas, \& K. Werner, ASP Conf. Ser., 288, 31

Werner, K., Rauch, T., Ringat, E., \& Kruk, J. W. 2012, ApJ, 753, L7

Wolff, B., Koester, D., Dreizler, S., \& Haas, S. 1998, A\&A, 329, 1045

Ziegler, M., Rauch, T., Werner, K., Köppen, J., \& Kruk, J. W. 2012, A\&A, 548, A109 


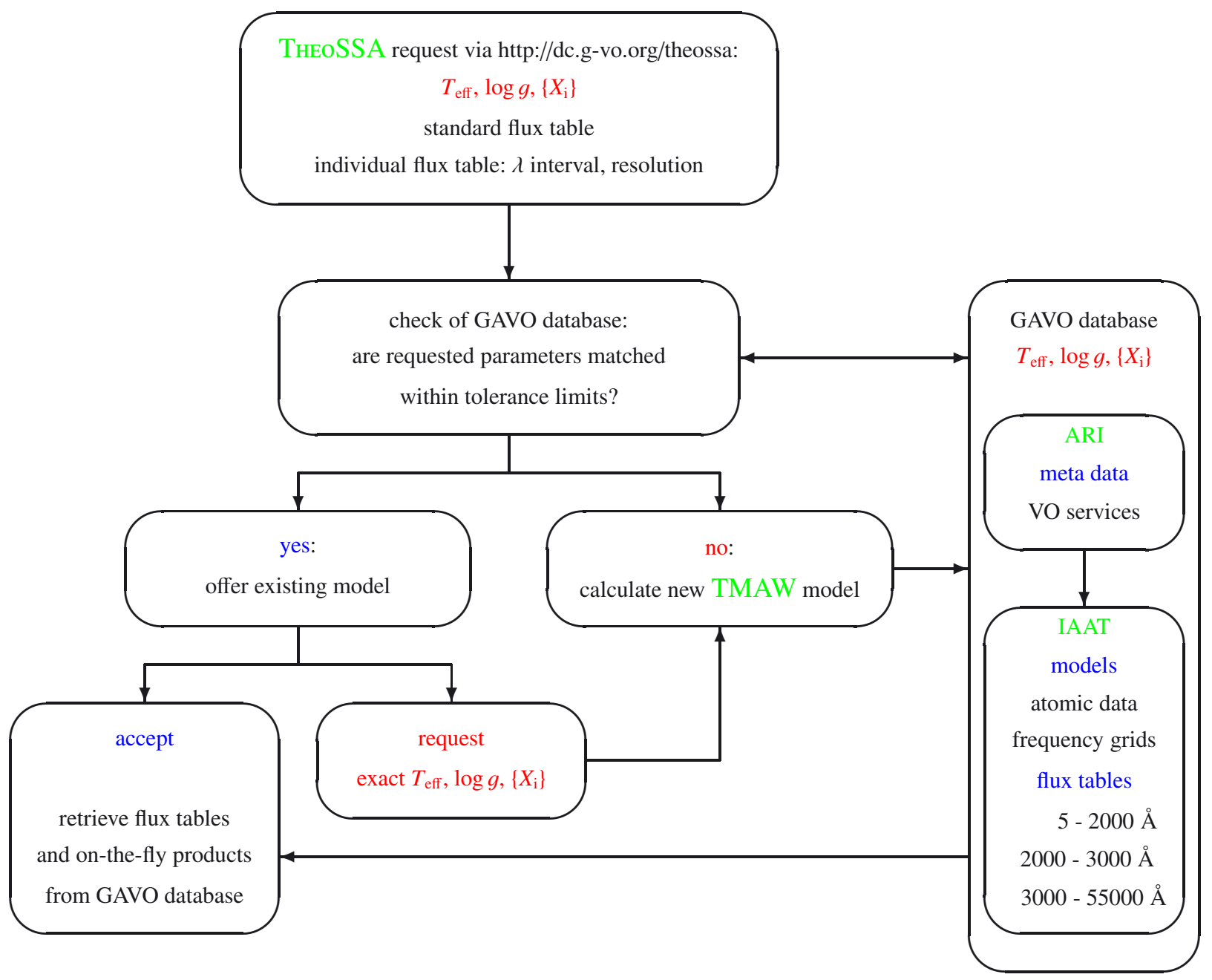

Fig. 1. Flow diagram of TheoSSA. The VO user sends an SED request to the GAVO database by entering the photospheric parameters. If a suitable model is available within the desired tolerance limits, it is offered as a results table. In case that the parameters are not exactly matched, the VO user may decide to calculate a model with the exact parameters. TMAW will start a model-atmosphere calculation at our institute's (IAAT) PC cluster then. Extended model grids make use of computer resources that are provided by AstroGrid-D. As soon as the model is converged, the VO user can retrieve the SED table from the GAVO database. 
T. Rauch et al.: TheoSSA: Establishing a database of synthetic stellar flux standards

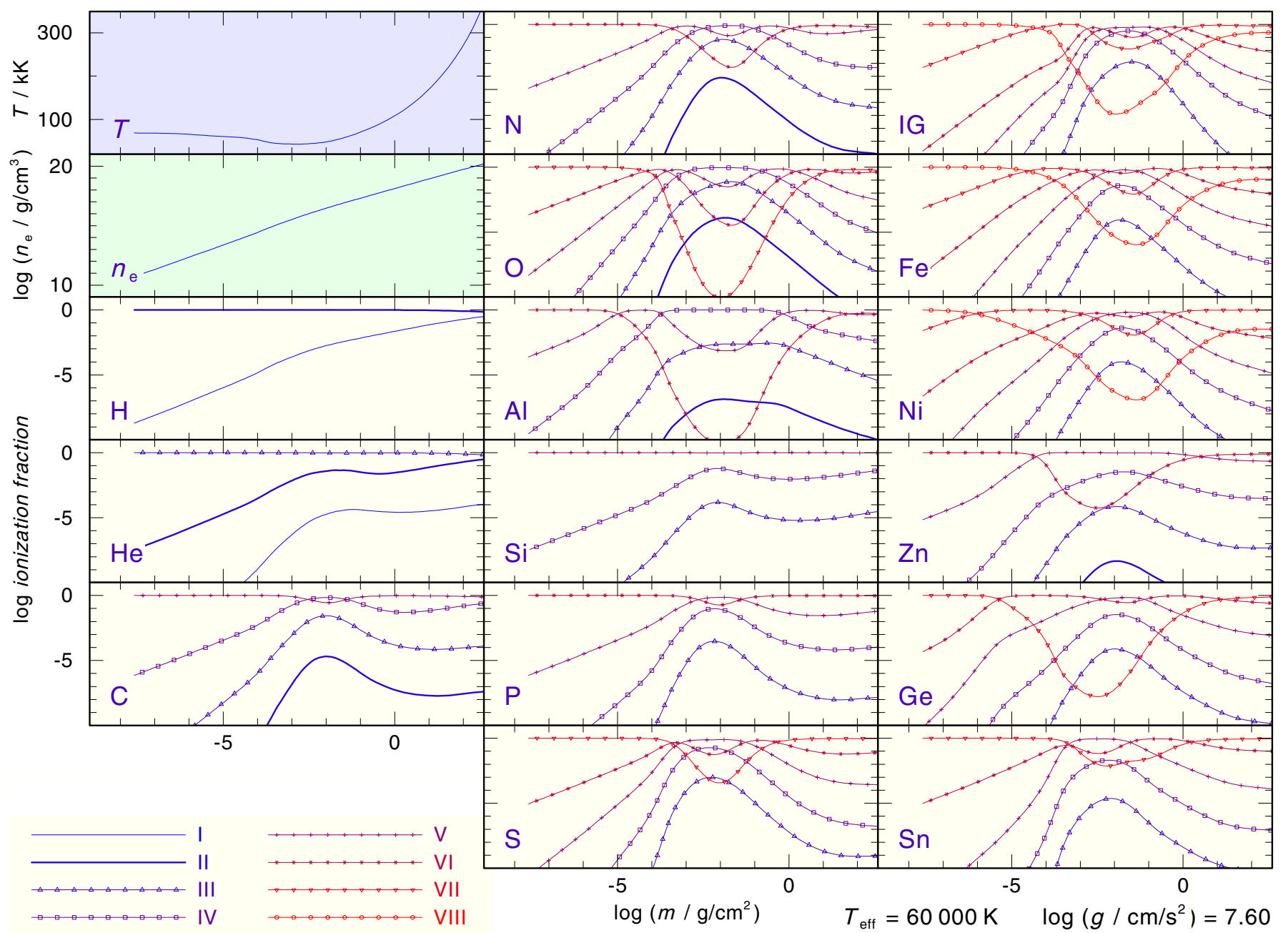

Fig. 6. Temperature and density structure and ionizations fractions of our model with $T_{\text {eff }}=60000 \mathrm{~K}$ and $\log g=7.60$. IG denotes a generic model atom consisting of $\mathrm{Ca}, \mathrm{Sc}, \mathrm{Ti}, \mathrm{V}, \mathrm{Cr}, \mathrm{Mn}$, and $\mathrm{Co}$. 
A\&A 560, A106 (2013)

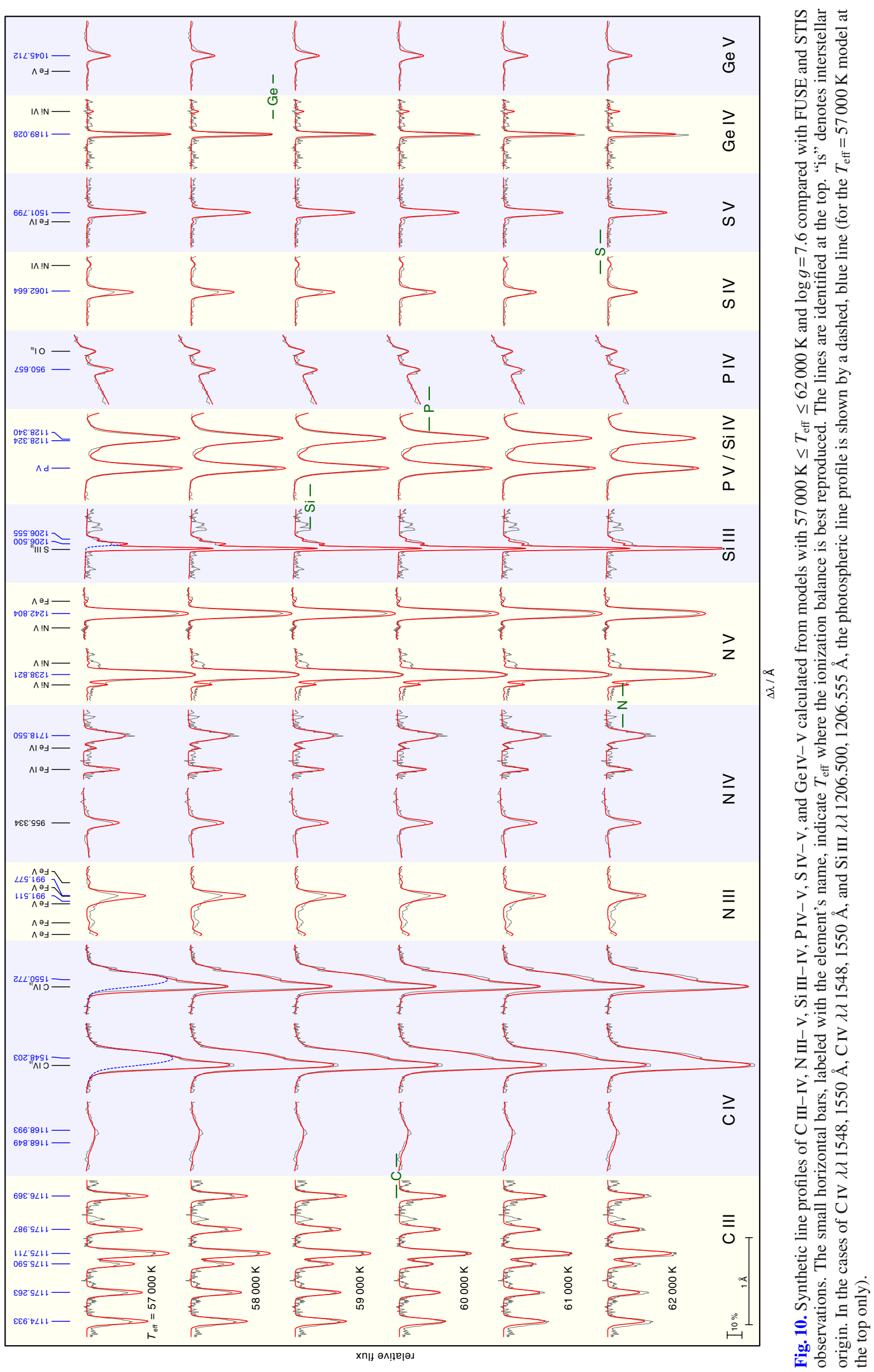


T. Rauch et al.: TheoSSA: Establishing a database of synthetic stellar flux standards
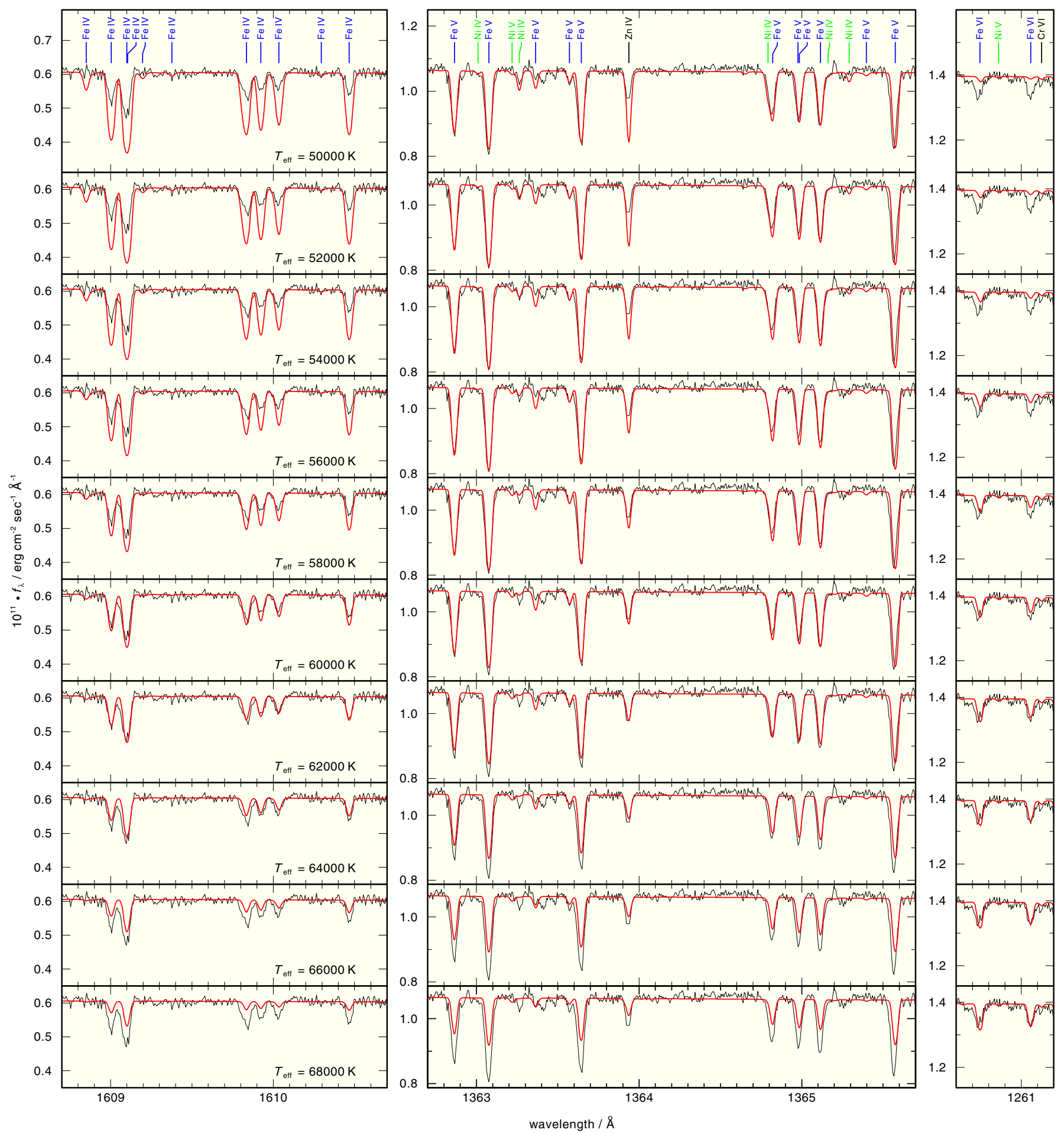

Fig. 11. Same as Fig. 10 for Fe IV-VI lines (from left to right panels, marked blue in the top panels) only. 
A\&A 560, A106 (2013)
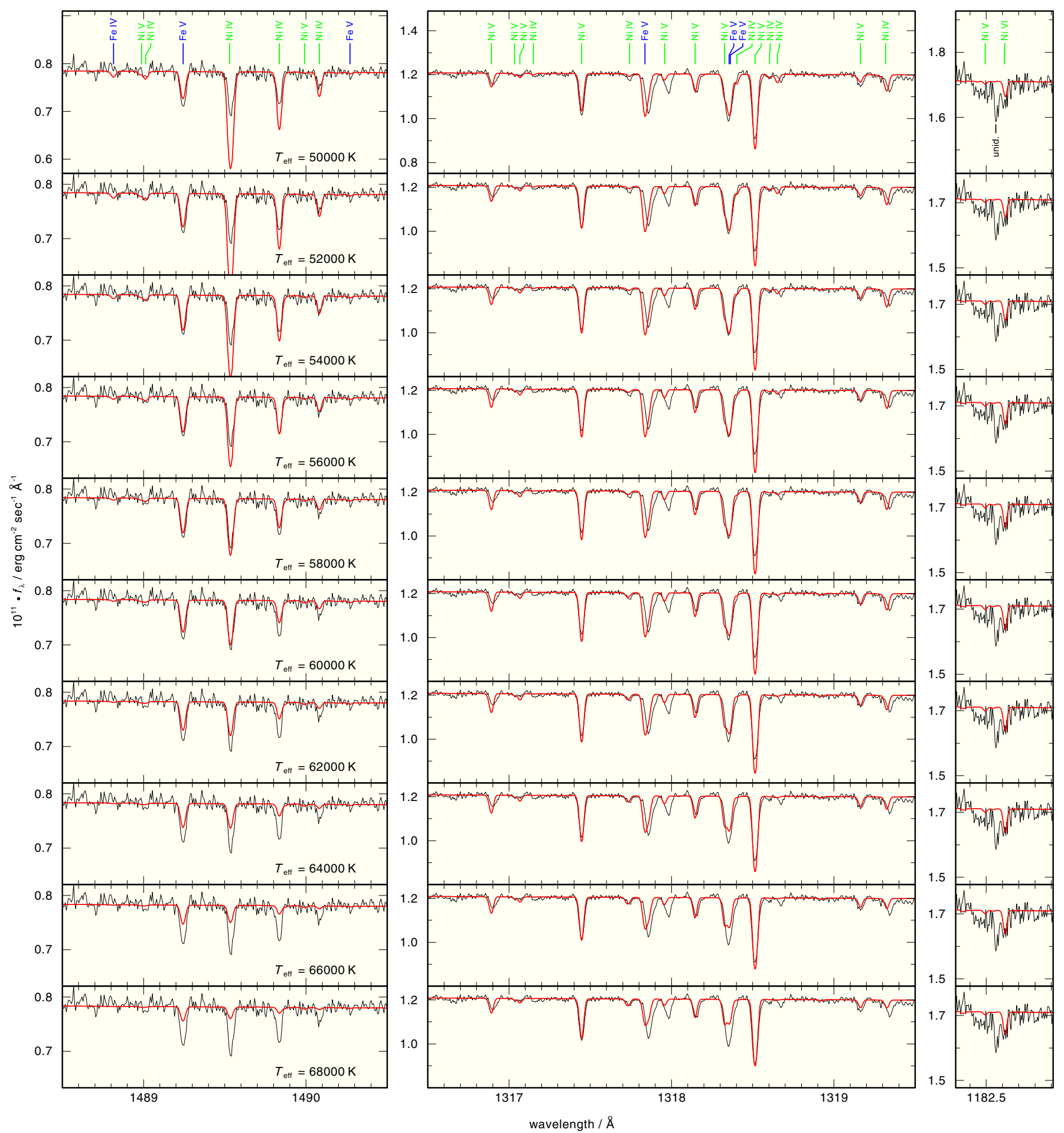

Fig. 12. Same as Fig. 10 for Ni IV-VI lines (from left to right panels, marked green in the top panels) only. 
T. Rauch et al.: TheoSSA: Establishing a database of synthetic stellar flux standards

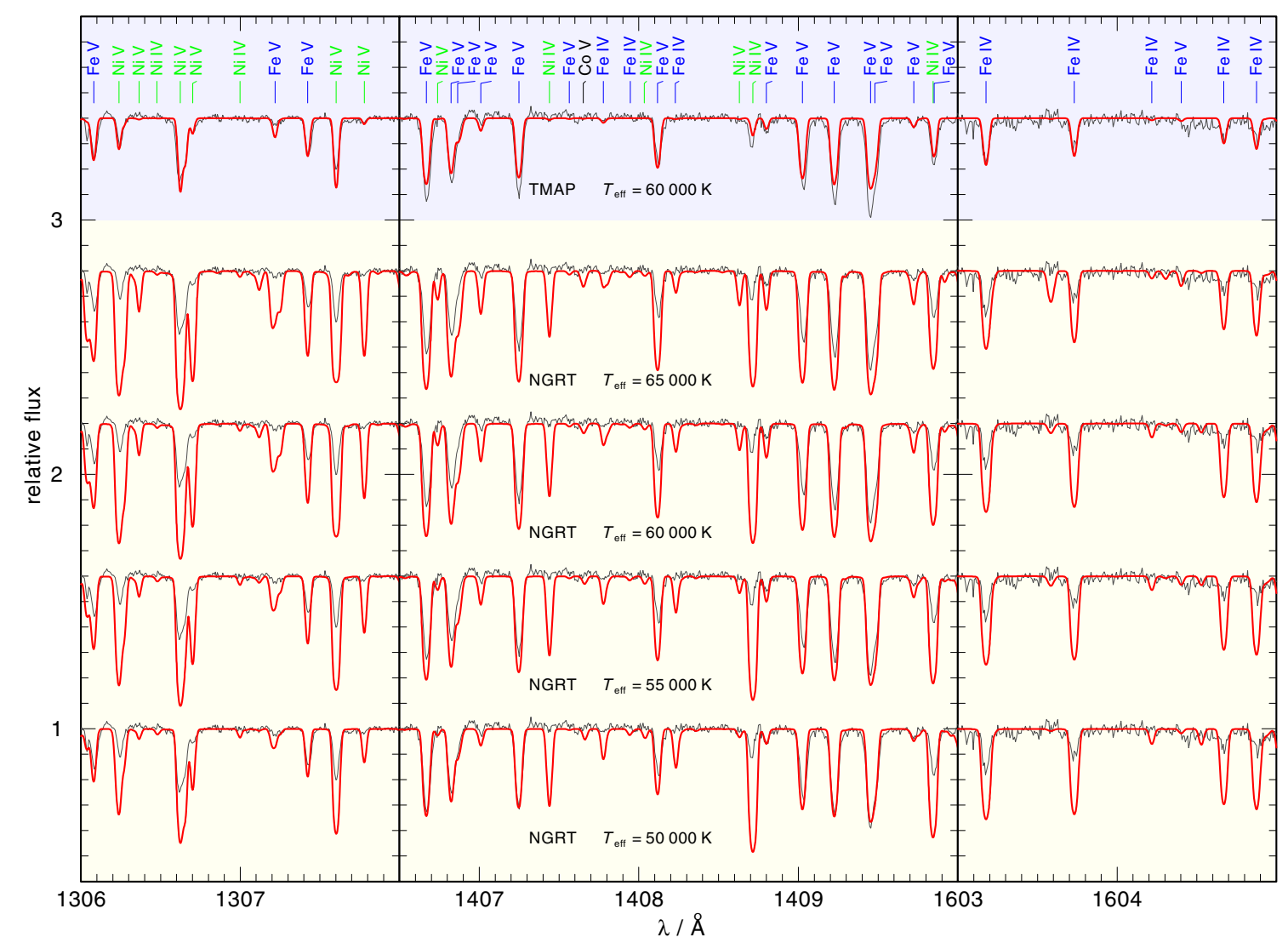

Fig. 23. Comparison of our model spectra (all calculated with $\log g=7.60)$ with the STIS observation. Top: final homogeneous model (TMAP). The stratified NGRT models have $T_{\text {eff }}=65000 \mathrm{~K}, 60000 \mathrm{~K}, 55000 \mathrm{~K}$, and $50000 \mathrm{~K}$ (from top to bottom). The lines in the models are marked. 
Table 2. $T_{\text {eff }}$ and $\log g$ from previous analyses of G191-B2B.

\begin{tabular}{|c|c|c|c|}
\hline $\begin{array}{l}T_{\text {eff }} \\
{[\mathrm{K}]} \\
\end{array}$ & $\begin{array}{l}\log g \\
{\left[\mathrm{~cm} / \mathrm{s}^{2}\right]}\end{array}$ & Reference & Method \\
\hline 61900 & $7.5^{a}$ & Shipman (1979) & LTE, pure H, optical colors \\
\hline $56788 \pm 3336$ & $5.95 \pm 0.04$ & Koester et al. (1979) & LTE, pure H, optical colors, ${ }^{b}$ \\
\hline $62250 \pm 3520$ & $7.55 \pm 0.35$ & Holberg et al. (1986) & LTE, pure $\mathrm{H}, \mathrm{H}$ I L $\alpha$ line \\
\hline $59250 \pm 2000$ & $7.50 \pm 0.10$ & $\begin{array}{l}\text { Kidder (1990) } \\
\text { cited by Holberg et al. (1991) }\end{array}$ & LTE, pure $\mathrm{H}, \mathrm{HI} \mathrm{H} \gamma$ and $\mathrm{H} \delta$ lines \\
\hline $61000_{-4000}^{+6000}$ & $8.00^{a}$ & Green et al. (1990) & LTE, H+He, extreme ultraviolet (EUV) continuum \\
\hline $61170_{-4230}^{+4030}$ & $8.00^{a}$ & Finley et al. (1990) & LTE, $\mathrm{H}+\mathrm{He}$, ultraviolet (UV) continuum \\
\hline $62250 \pm 1000$ & $8.00^{a}$ & Finley et al. (1990) & LTE, $\mathrm{H}+\mathrm{He}, \mathrm{H}$ I L $\alpha$ line \\
\hline 62250 & 7.55 & Vennes et al. (1991) & NLTE, $\mathrm{H}+\mathrm{HeCNSi}$, UV spectrum \\
\hline $53500 \pm 500$ & & Koester \& Finley (1992) & LTE, $\mathrm{H}+\mathrm{He}^{c}, \mathrm{UV}$ continuum \\
\hline $60500 \pm 900$ & $7.50 \pm 0.05$ & Vidal-Madjar et al. (1994) & LTE, H+He, H I Balmer lines \\
\hline $57900 \pm 1500$ & $7.50^{a}$ & Dupuis et al. (1995) & NLTE, pure H, EUV continuum ${ }^{d}$ \\
\hline $54000 \pm 800$ & $7.50^{a}$ & Dupuis et al. (1995) & NLTE, H+CNOFe, EUV continuum ${ }^{d}$ \\
\hline $60500 \pm 1000$ & 7.5 & Lanz et al. (1996) & LTE, H+HeC, H I Balmer lines \\
\hline $56000 \pm 1000$ & 7.5 & Lanz et al. (1996) & NLTE, H+HeC, H I Balmer lines \\
\hline $55200 \pm 1000$ & 7.5 & Lanz et al. (1996) & NLTE, $\mathrm{H}+\mathrm{HeCFe}$, H I Balmer lines \\
\hline $64000 \pm 1000$ & $7.64 \pm 0.06$ & Vennes et al. (1996) & LTE, pure H, H I Lyman lines \\
\hline $57900 \pm 1500$ & 7.5 & Vennes et al. (1996) & LTE, pure H, extreme UV (EUV) continuum ${ }^{d}$ \\
\hline $64100 \pm 700$ & $7.69 \pm 0.04$ & Vennes et al. (1996) & LTE, H+CNOFe, H I Balmer lines \\
\hline $52600 \pm 800$ & $7.53 \pm 0.07$ & Vennes et al. (1996) & LTE, H+CNOFe, H I Lyman lines \\
\hline $54000 \pm 800$ & 7.5 & Vennes et al. (1996) & LTE, $\mathrm{H}+\mathrm{CNOFe}$, EUV continuum ${ }^{d}$ \\
\hline $61193 \pm 241$ & $7.49 \pm 0.01$ & Finley et al. (1997) & LTE, H-Ni, H I Balmer lines \\
\hline $59160_{-1070}^{+1270}$ & $7.36_{-0.07}^{+0.08}$ & Barstow et al. (1998) & NLTE, pure H, H I Balmer lines \\
\hline $59190_{-820}^{+1400}$ & $7.36_{-0.07}^{+0.07}$ & Barstow et al. (1998) & NLTE, H+He, H i Balmer lines \\
\hline $59060_{-1090}^{+1130}$ & $7.36_{-0.07}^{+0.08}$ & Barstow et al. (1998) & NLTE, $\mathrm{H}+\mathrm{He}+$ heavy-metal poor, H I Balmer lines \\
\hline $53840_{-160}^{+400}$ & $7.38_{-0.08}^{+0.07}$ & Barstow et al. (1998) & NLTE, H+He + heavy-metal rich, H I Balmer lines \\
\hline $52920 \pm 350$ & $7.36 \pm 0.03$ & Barstow et al. (1998) & NLTE, H+He + heavy-metal rich, H I Lyman lines ${ }^{e}$ \\
\hline 56000 & 7.6 & Wolff et al. (1998) & LTE + NLTE, H+CNOSiFeNi, EUV continuum ${ }^{d}$ \\
\hline 56000 & 7.6 & Dreizler \& Wolff (1999) & NLTE, H+CNOSiFeNi, diffusion model, EUV to optical \\
\hline $54600 \pm 200$ & $7.60 \pm 0.02$ & Barstow et al. (2001) & NLTE, H+HeCNOSiFeNi, H I Balmer lines \\
\hline $52930 \pm 3600$ & $7.16 \pm 0.2$ & Barstow et al. (2001) & NLTE, $\mathrm{H}+\mathrm{HeCNOSiFeNi,} \mathrm{H} \mathrm{I} \mathrm{Lyman} \mathrm{lines}{ }^{f}$ \\
\hline $53180 \pm 530$ & $7.43 \pm 0.04$ & Barstow et al. (2001) & NLTE, $\mathrm{H}+\mathrm{HeCNOSiFeNi}$, H I Lyman lines ${ }^{e}$ \\
\hline 56000 & 7.59 & Schuh et al. (2002) & NLTE, H+HeCNOSiFeNi, diffusion model, EUV continuum ${ }^{d}$ \\
\hline 54000 & 7.5 & Holberg et al. (2003) & NLTE, metal lines \\
\hline $58865 \pm 706$ & $7.57 \pm 0.038$ & Lajoie \& Bergeron (2007) & $\mathrm{NLTE}^{g}$, pure H, H i Balmer lines \\
\hline $60680 \pm 15000$ & $7.57^{h}$ & Lajoie \& Bergeron (2007) & $\mathrm{NLTE}^{g}$, pure H, H I Lyman lines ${ }^{i}$ \\
\hline $57414 \pm 4700$ & $7.57^{h}$ & Lajoie \& Bergeron (2007) & $\mathrm{NLTE}^{g}$, pure H, V-normalization method \\
\hline $61980 \pm 514$ & $7.56 \pm 0.04$ & Allende Prieto et al. (2009) & NLTE, $\mathrm{H}^{j}, \mathrm{H}$ I Balmer lines \\
\hline $60920 \pm 993$ & $7.55 \pm 0.05$ & Gianninas et al. (2011) & NLTE, H+CNO, H I Balmer lines ${ }^{k}$ \\
\hline
\end{tabular}

Notes. ${ }^{(a)}$ Assumed $\log g$ value. ${ }^{(b)}$ The authors note that the results are extrapolated from their model grid. ${ }^{(c)}$ Stratified model, hydrogen-layer mass between $6 \times 10^{-15}$ and $8 \times 10^{-15} M_{\odot}{ }^{(d)}$ Extreme Ultraviolet Explorer (EUVE, http://heasarc.gsfc.nasa.gov/docs/euve/euve.html) observations. ${ }^{(e)}$ Orbiting and Retrievable Far and Extreme Ultraviolet Spectrometer (ORFEUS, http://www.uni-tuebingen.de/en/4221) and FUSE observations. ${ }^{(f)}$ Hopkins Ultraviolet Telescope (HUT, http://praxis.pha.jhu.edu/) observations. ${ }^{(g)}$ International Ultraviolet Explorer (IUE) observations. ${ }^{(h)}$ Adopted from their optical solution. ${ }^{(i)}$ Models described in Liebert et al. (2005). ${ }^{(j)}$ The authors note that $T_{\text {eff }}$ may be overestimated by $\approx 6000 \mathrm{~K}$ because their pure-H models are inappropriate due to the photospheric metal content. ${ }^{(k)}$ New H I Stark linebroadening tables from Tremblay \& Bergeron (2009). 
T. Rauch et al.: TheoSSA: Establishing a database of synthetic stellar flux standards

Table 3. Abundances of photospheric trace elements in G191-B2B from previous analyses.

\begin{tabular}{llll}
\hline \hline Element & & \multicolumn{2}{l}{ Log mass fraction } \\
\cline { 1 - 1 } $\mathrm{He}$ & & $-4.2 \pm 0.1^{a}$ & $-4.4^{i}$ \\
$\mathrm{C}$ & & $-4.4 \pm 0.3^{b}$ & \\
& & $-4.6 \pm 0.3^{c}$ & $-5.6^{i}$ \\
$\mathrm{~N}$ & & $-4.6 \pm 0.3^{b}$ & \\
& & $-5.3 \pm 0.4^{d}$ & $-5.9^{i}$ \\
$\mathrm{O}$ & & $-4.8 \pm 0.3^{b}$ & \\
& & $-4.8 \pm 0.3^{e}$ & $-4.6^{i}$ \\
$\mathrm{Mg}$ & & & $<-5.6^{i}$ \\
$\mathrm{Al}$ & & & $-5.1^{i}$ \\
$\mathrm{Si}$ & & $-5.1 \pm 0.4^{d}$ & $-5.0^{i}$ \\
& & $-5.1 \pm 0.5^{c}$ & \\
$\mathrm{P}$ & & $-5.0 \pm 0.3^{b}$ & \\
$\mathrm{~S}$ & & $-6.2 \pm 0.2^{c}$ & \\
$\mathrm{Cl}$ & & $-5.2 \pm 0.5^{c}$ & \\
$\mathrm{Cr}$ & & $<-7.0^{b}$ & \\
$\mathrm{Mn}$ & & & $<-6.3^{i}$ \\
$\mathrm{Fe}$ & & $-3.8 \pm 0.3^{d}$ & $<-6.3^{i}$ \\
& & $-3.4 \pm 0.4^{f}$ & \\
& & $-3.3 \pm 0.3^{b}$ & \\
$\mathrm{Co}$ & & & $<-6.2^{i}$ \\
$\mathrm{Ni}$ & & $-4.2 \pm 0.5^{g}$ & $-4.4^{i}$ \\
& $-4.2 \pm 0.4^{f}$ & \\
$\mathrm{Ge}$ & $-3.9 \pm 0.3^{b}$ & \\
$\mathrm{Sn}$ & $-6.1 \pm 0.2^{h}$ & \\
\hline
\end{tabular}

Notes. He abundance assumed, no abundance uncertainties given. ${ }^{(a)}$ Cruddace et al. (2002); ${ }^{(b)}$ Lanz et al. (1996); ${ }^{(c)}$ Vennes et al. (1996); (d) Vidal-Madjar et al. (1994); ${ }^{(e)}$ Chayer et al. (1996); ${ }^{(f)}$ Werner \& Dreizler (1994); ${ }^{(g)}$ Holberg et al. (1994); ${ }^{(h)}$ Vennes et al. (2005); ${ }^{(i)}$ Holberg et al. (2003). 
Table 4. Statistics of our model atoms.

\begin{tabular}{|c|c|c|c|c|}
\hline Ion & NLTE levels & LTE levels & Lines & Sample lines \\
\hline H I & 14 & 2 & 91 & \\
\hline II & 1 & - & - & \\
\hline He I & 29 & 74 & 69 & \\
\hline II & 16 & 16 & 120 & \\
\hline III & 1 & - & - & \\
\hline C II & 1 & 45 & 0 & \\
\hline III & 44 & 23 & 190 & \\
\hline IV & 54 & 4 & 295 & \\
\hline $\mathrm{V}$ & 1 & 0 & 0 & \\
\hline $\mathrm{N}$ II & 1 & 246 & 0 & \\
\hline III & 34 & 32 & 129 & \\
\hline IV & 90 & 4 & 546 & \\
\hline $\mathrm{V}$ & 54 & 8 & 297 & \\
\hline VI & 1 & 0 & 0 & \\
\hline O II & 1 & 46 & 0 & \\
\hline III & 72 & 0 & 322 & \\
\hline IV & 38 & 56 & 173 & \\
\hline $\mathrm{V}$ & 76 & 50 & 472 & \\
\hline VI & 54 & 8 & 291 & \\
\hline VII & 1 & 0 & 0 & \\
\hline $\mathrm{Al}$ II & 1 & 4 & 0 & \\
\hline III & 7 & 29 & 10 & \\
\hline IV & 6 & 183 & 3 & \\
\hline $\mathrm{V}$ & 6 & 223 & 4 & \\
\hline VI & 1 & 0 & 0 & \\
\hline Si III & 17 & 17 & 28 & \\
\hline IV & 16 & 7 & 44 & \\
\hline $\mathrm{v}$ & 1 & 0 & 0 & \\
\hline P III & 3 & 7 & 0 & \\
\hline IV & 21 & 30 & 9 & \\
\hline $\mathrm{V}$ & 18 & 7 & 12 & \\
\hline VI & 1 & 0 & 0 & \\
\hline S III & 1 & 230 & 0 & \\
\hline IV & 17 & 83 & 32 & \\
\hline $\mathrm{V}$ & 39 & 71 & 107 & \\
\hline VI & 25 & 12 & 48 & \\
\hline VII & 1 & 0 & 0 & \\
\hline $\mathrm{Fe}$ III & 7 & 0 & 25 & 537689 \\
\hline IV & 7 & 0 & 25 & 3102371 \\
\hline $\mathrm{v}$ & 7 & 0 & 25 & 3266247 \\
\hline VI & 7 & 0 & 33 & 991935 \\
\hline VII & 7 & 0 & 39 & 200455 \\
\hline VIII & 1 & 0 & 0 & 0 \\
\hline Ni III & 7 & 0 & 22 & 1033920 \\
\hline IV & 7 & 0 & 25 & 2512561 \\
\hline $\mathrm{V}$ & 7 & 0 & 27 & 2766664 \\
\hline VI & 7 & 0 & 27 & 7408657 \\
\hline VII & 7 & 0 & 33 & 4195381 \\
\hline VIII & 1 & 0 & 0 & 0 \\
\hline IG III & 1 & 0 & 0 & 0 \\
\hline IV & 7 & 0 & 25 & 1579918 \\
\hline V & 7 & 0 & 23 & 2230921 \\
\hline VI & 7 & 0 & 25 & 1455521 \\
\hline VII & 7 & 0 & 24 & 1129512 \\
\hline VIII & 1 & 0 & 0 & 0 \\
\hline
\end{tabular}

Notes. IG denotes a generic model atom consisting of Ca, Sc, Ti, V, Cr, Mn, and Co. "Sample lines" are individual Kurucz lines that are sampled to superlines for Ca-Ni (Rauch \& Deetjen 2003). 
T. Rauch et al.: TheoSSA: Establishing a database of synthetic stellar flux standards

Table 4. continued.

\begin{tabular}{cccccc}
\hline \hline & Ion & NLTE levels & LTE levels & Lines & Sample lines \\
\hline Zn II & 1 & 5 & 0 & \\
III & 2 & 10 & 0 & \\
IV & 31 & 0 & 87 & \\
V & 5 & 15 & 0 & \\
VI & 1 & 0 & 0 & \\
Ge III & 1 & 15 & 0 & \\
IV & 8 & 1 & 8 & \\
V & 85 & 0 & 878 & \\
VI & 11 & 25 & 0 & \\
VII & 1 & 0 & 0 & \\
Sn III & 3 & 18 & 2 & \\
IV & 6 & 4 & 1 & \\
V & 5 & 4 & 0 & \\
VI & 6 & 0 & 0 & \\
VII & 1 & 0 & & 32411752 \\
\hline Total & 70 & 1038 & 1614 & 4646 & \\
\hline
\end{tabular}


A\&A 560, A106 (2013)

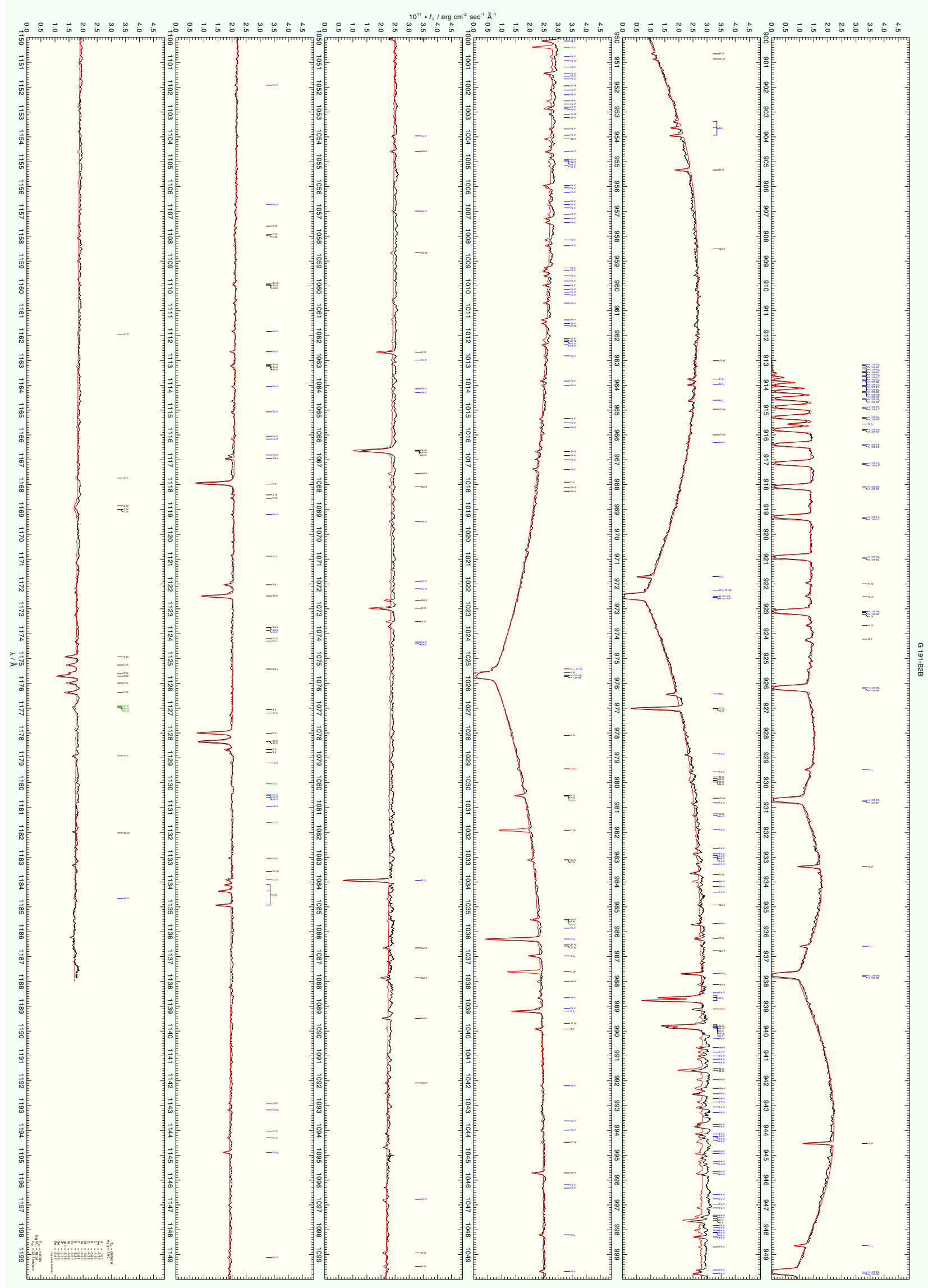

Fig. A.1. Comparison of the FUSE observation with our final model. Stellar and interstellar lines are identified. "is" denotes interstellar origin, "unid." denotes unidentified lines. 
T. Rauch et al.: TheoSSA: Establishing a database of synthetic stellar flux standards

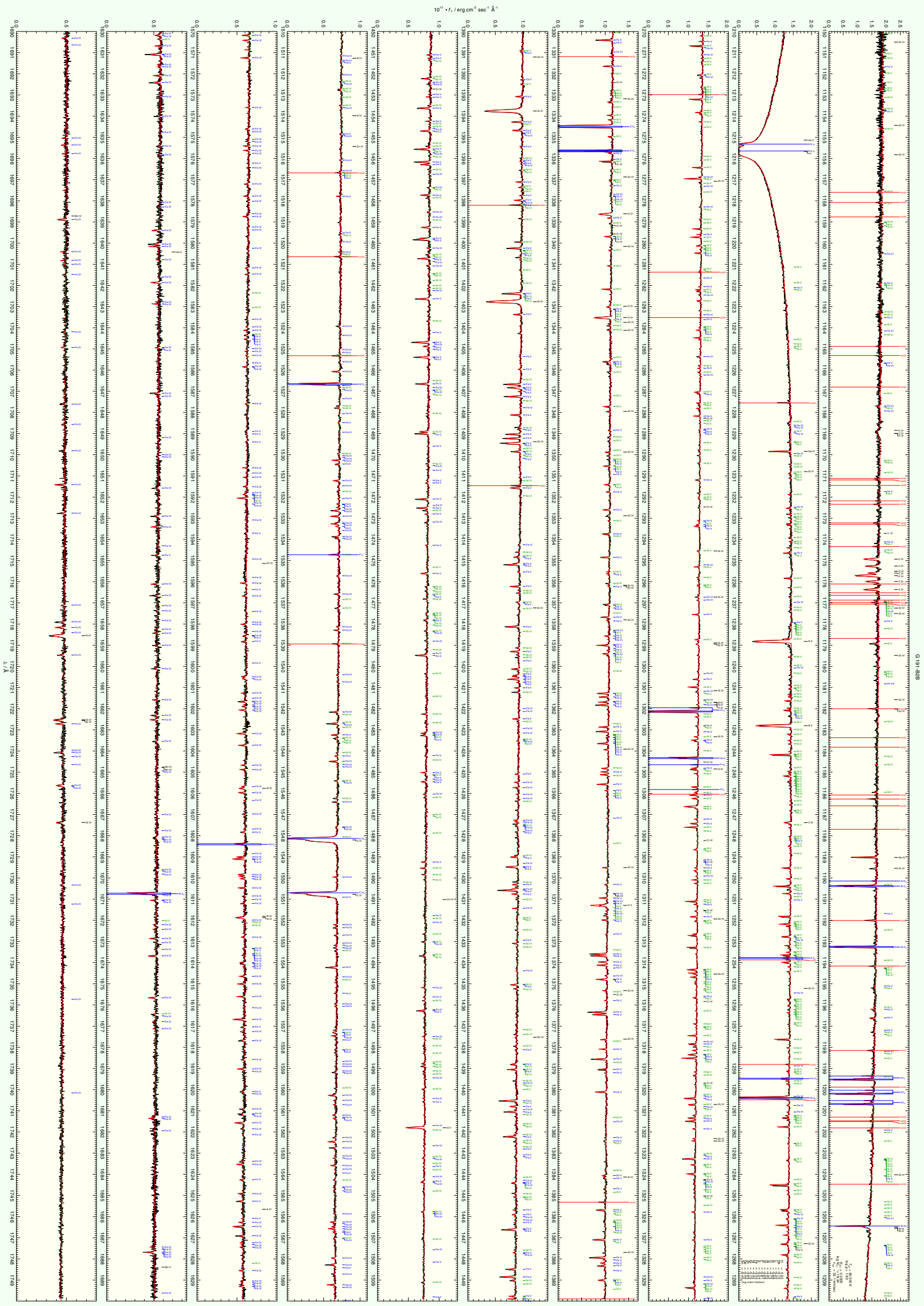

Fig. A.2. Comparison of a section of the STIS observation with our final model. Stellar and interstellar lines are identified. "is" denotes interstellar origin, "unid." denotes unidentified lines. 